\title{
Mathematical Study of Pattern Formation Accompanied by Heterocyst Differentiation in Multicellular Cyanobacterium
}

Jun-ichi Ishihara ${ }^{1,2}$, Masashi Tachikawa ${ }^{2}$, Hideo Iwasaki ${ }^{1}$, Atsushi Mochizuki ${ }^{2}$

${ }^{1}$ Department of Electrical Engineering \& Bioscience, Waseda University, TWIns, Shinjuku 162-8480, Japan

${ }^{2}$ Theoretical Biology Laboratory, RIKEN, Wako 351-0198, Japan

Running Headline: mathematical study of heterocyst pattern formation

Corresponding Author: Jun-ichi Ishihara, Atsushi Mochizuki

Address: $\quad$ Theoretical Biology Laboratory, RIKEN, Wako 351-0198, Japan

E-mail: $\quad$ jishihara@aoni.waseda.jp, mochi@ @ $\quad$ riken.jp

Telephone: $\quad+81-48-467-8422$

Facsimile: $\quad+81-48-462-1709$ 


\section{Abstract}

The filamentous cyanobacterium, Anabaena sp. PCC 7120, is one of the simplest models of a multicellular system showing cellular differentiation. In nitrogen-deprived culture, undifferentiated vegetative cells differentiate into heterocysts at $\sim 10$-cell intervals along the cellular filament. As undifferentiated cells divide, the number of cells between heterocysts (segment length) increases, and a new heterocyst appears in the intermediate region.

To understand how the heterocyst pattern is formed and maintained, we constructed a one-dimensional cellular automaton (CA) model of the heterocyst pattern formation. The dynamics of vegetative cells is modeled by a stochastic transition process including cell division, differentiation and increase of cell age (maturation). Cell division and differentiation depend on the time elapsed after the last cell division, the "cell age". The model dynamics was mathematically analyzed by a two-step Markov approximation. In the first step, we determined steady state of cell age distribution among vegetative cell population. In the second step, we determined steady state distribution of segment length among segment population. The analytical solution was consistent with the results of numerical simulations. We then compared the analytical solution with the experimental data, and quantitatively estimated the immeasurable intercellular kinetics. We found that differentiation is initially independent of cellular maturation, but becomes dependent on maturation as the pattern formation evolves. Our mathematical model and analysis enabled us to quantify the internal cellular dynamics at various stages of the heterocyst pattern formation. 


\section{Keywords}

cell lineage analysis; cellular maturation; cellular automaton model; Markov process; estimation

\section{Introduction}

In nitrogen-rich environments, the multicellular (filamentous) cyanobacterium, Anabaena sp. PCC 7120, comprises a one-dimensional filament of vegetative cells. Under nitrogen deprivation, some of the vegetative cells differentiate into heterocysts. Vegetative cells and heterocysts are specialized for photosynthesis and nitrogen fixation, respectively (Zhang et al., 2006; Kumar et al., 2009). Heterocyst differentiation occurs at approximately 10-cell intervals along the filament.

Heterocysts supply the surrounding vegetative cells with fixed nitrogen products and receive carbohydrate products in return. This metabolic cooperation between the two cell types is essential to filament growth under nitrogen deprived conditions (Zhang et al., 2006; Kumar et al., 2009; Cumino et al., 2007). During diazotrophic growth, intercellular fixed nitrogen is transported by periplasmic diffusion along the Anabaena filament (Flores et al., 2006; Mariscal et al., 2006; Walsby et al., 2007).

Once formed, a heterocyst cannot divide or dedifferentiate into a vegetative cell (Zhang et al., 2006; Golden and Yoon, 2003). As the number of vegetative cells increases by the cell division, a new heterocyst appears approximately midway between two older heterocysts. As a simple self-organizational process, heterocyst pattern formation in Anabaena is an important model system for investigating multicellular development (Fig. 1). We consider that heterocyst position and frequency are regulated 
to optimize the distribution of fixed nitrogen compounds within a long filament for optimal growth. Because the differentiation is necessary but costly, the number of vegetative cells between heterocysts must be optimized for steady-state growth in nitrogen deprived conditions. Indeed, a gradual increase has been reported in the mean number of vegetative cells between heterocysts as the pattern formation proceeds (Yoon and Golden, 2001). In our observed culture system, the number of vegetative cells between heterocysts also established a phenotypic steady state.

Heterocyst pattern formation is consistent with the idea that highly concentrated nitrogen fixation products produced by developing/mature heterocysts suppress the differentiation of neighboring cells (Yoon and Golden, 2001; Wilcox et al., 1973a, 1973b; Wolk, 1975). This suggests that differentiation is controlled by nitrogen availability. On the other hands, genetic dissection has identified a gene involved in heterocyst pattern formation, which collectively encode a lateral inhibition system. Heterocysts produce a polypeptide PatS, which is likely to diffuse through the periplasm and inhibit the differentiation of neighboring cells (Yoon and Golden, 1998; Callahan and Buikema, 2001; Wu et al., 2004). It appears that nitrogen compounds and PatS are the primary regulators of heterocyst pattern formation. However, their spatiotemporal cooperation within the filament remains poorly understood, because the chemical gradients of such small molecules cannot be detected by existing experimental techniques. In the same way, several genes that activate differentiation have been also identified. Especially, the hetR gene is expressed during the initial differentiation phase, and its protein product, HetR, activates its own transcription (Buikema and Haselkorn, 1991; Black et al., 1993; Zhou et al., 1998). HetR also activates the expression of patS encoding the PatS polypeptide, inhibiting HetR's function (Huang et al., 2004). While 
these internal and external signals are necessary for controlling cell fate and heterocyst pattern formation (Golden and Yoon, 2003), the coordination of their roles is not quantitatively understood. Most previous studies are based on statistical analysis of filaments collected at different times from liquid cultures. Such studies cannot reveal the temporal changes in molecular activity.

To better understand the spatiotemporal dynamics of heterocyst pattern formation, we observed morphological changes, het $R$ gene expression and phycobilisome fluorescence transition in a whole filament grown under the microscope (Asai et al., 2009). We analyzed the time-lapse data and integrated them into a cell lineage, representing the timing and positions of cell division and differentiation after nitrogen depletion. Previously, we found that:

i) differentiation is coupled to cell division (cell cycle),

ii) Vegetative cells are more likely to differentiate into heterocysts at earlier times of heterocyst pattern formation.

These findings suggest that the couplings of cell division and (or) differentiation to the cell cycle phase vary during the heterocyst pattern formation. However, we did not quantitatively validate this suggestion.

In the present study, we investigated the dependency of cell division/differentiation on cellular maturation. To this end, we constructed a mathematical model of the cellular maturation dynamics. By comparing the model results with the experimental results, we linked the maturation dynamics to heterocyst pattern formation. First, we reviewed and reanalyzed the cell lineage obtained from the time-lapse images (Asai et al., 2009). These data provided the temporal patterns of cell division and differentiation along the filament. Moreover, we analyzed the temporal 
progress of heterocyst pattern formation under nitrogen-deprived conditions by culturing many Anabaena filaments. We then developed a one-dimensional cellular automaton (CA) model of the intercellular dynamics. The modeled cell division and differentiation depend on time elapsed after the last cell division, here referred to as "cell age", which corresponds to the maturation level after cell division.

We observed different heterocyst patterns at earlier and later time after nitrogen depletion. From the cell lineage analysis, we assumed that the differentiation occurs independently on cellular maturation in earlier timing. Under this assumption, the generated patterns were similar to the observed patterns at earlier and later times during the pattern formation. Thus, we concluded that the different dependencies of differentiation on the cell age switch the heterocyst pattern between earlier and later times.

Using data from the cell lineage analysis and time-lapse images, we constructed a mathematical model that reflects the cellular state dynamics over the whole filament. In the situation that the gene network and metabolism crosstalk has not been studied sufficiently, our mathematical model based on cellular dynamics suggests the internal dynamics analytically. 


\section{Results}

\subsection{Analysis of cell lineage obtained from time-lapse monitoring video}

In our previous study (Asai et al., 2009), we cultured a mutant fluorescent reporter strain harboring a PhetR::gfp reporter cassette, termed SRhetR-1Gn, under the microscope and observed the heterocyst pattern formation of four individual filaments. In the present study, we studied the spatiotemporal patterns of cell division and differentiation in a single filament, again by analyzing the cell lineage obtained from the time-lapse monitoring video (Movie 2A and Figure S2A in Asai et al., 2009). At the time of nitrogen depletion (time $=0$ ), this filament consisted of 24 vegetative cells, expanding to 468 cells (including 39 heterocysts) at 72 hours after nitrogen depletion (abbreviated as a.n.d.).

First, we analyzed the temporal pattern of cell division in the nitrogen-starved cells. By calculating the duration time after the latest cell division among the vegetative cells in the focal filament, we determined the duration distributions of the vegetative cells. Cell division was marked by a clear visible septum between the daughter cells (Sakr et al., 2006a, 2006b). For the cell which had not divided, we defined the duration time as hours after nitrogen depletion. We observed only the limited number of cell division before 30 hours a.n.d., and we calculated the distributions of duration time at 30 hours a.n.d., and thereafter at five-hourly intervals up to 70 hours a.n.d. (i.e., at 30, $35,40,45,50,55,60,65,70$ hours a.n.d.). Some of these distributions are shown in Fig.

2A. The pattern of the duration distribution alters over time and eventually appears to equilibrate. Several statistical analyses revealed the equilibrium time as approximately 35 hours a.n.d. Fig. 2B shows the temporal changes in principal components of the 
duration distributions on the PC1-PC2 plane. The PC2 score values are Gaussian distributed $(p>0.1$; two-sided Kolmogorov-Smirnov test for the Gaussian null hypothesis). The PC2 score for 30 hours a.n.d. is not included in the two-sided $95 \%$ confidence interval. We also directly evaluated the convergence of the distribution by conducting two-sample two-sided Kolmogorov-Smirnov tests (Table C1). Again, the distributions were significantly different between 30 and 35 hours a.n.d. $(p<0.01)$, and invariant after 35 hours a.n.d. (up to 70 hours a.n.d.). We considered that the regulatory mechanism of the cell cycle was initially disturbed by nitrogen depletion, but the effect of disturbance disappeared after 35 hours a.n.d.

We also calculated cell cycle length directly as the difference between two time points of sequential cell divisions along the cell lineage. We refer to the cumulative number of cell divisions after nitrogen depletion as the "generation time". All cells in the filament were regarded as the cells in the generation 0 at the time of nitrogen depletion. Cell division increases the generation number of daughter cells by one step. For example, the filament at 72 hours a.n.d. contained 237 cells after the fourth cell division (generation 4) and 210 cells after the fifth cell division (generation 5). Fig. 3A shows the distributions of the cell cycle length through the generations 0-4. Because all cells had divided twice after 35 hours a.n.d., it was expected that the disturbance to regulation of cell division disappears until the end of twice cell divisions. To confirm this, we analyzed the distributions of the cell cycle length in the generations 1-4. Fig. 3B shows the change in principal components of the distributions of cell cycle length on the PC1-PC2 plane. The distributions of cell cycle length converge after the generation 2 ( $p>0.9$; two-sample two-sided Kolmogorov-Smirnov test, see Table C2). Therefore, we summed the number of cells at each cell cycle length for the generations 2-4, and 
normalized the results by the number of cells summed over the three generations. The steady-state distribution of cell cycle length is shown in Fig. 3C.

Next, we analyzed the temporal pattern of differentiation. Differentiation is characterized by onset of cell expansion and reduction of phycobilisome fluorescence (Zhang et al., 2006). The first heterocyst appeared at 63 hours a.n.d., considerably later than the time at which the putative disturbance in cell cycle had already disappeared. In our previous investigation, the frequency of cells expressing the hetR gene was higher during earlier than later times of heterocyst pattern formation (Asai et al., 2009), suggesting that the differentiation mechanism alters at later time. Thus, we distinguished two stages; the early stage (defined as 63-65 hours a.n.d.) and the later stage (69- hours a.n.d.). This classification is reapplied in the present study.

In the following analysis, we examined the heterocyst pattern formations at both stages. To characterize the heterocyst pattern, we refer to an alignment of vegetative cells enclosed by two heterocysts as a "segment". In our terminology, the number of vegetative cells in a segment is a "segment length". We analyzed and compared distributions of segment length, which reflects the mechanism of differentiation at each stage. Newly, we cultured filaments of the PhetR::gfp reporter strain under the previously-described conditions (Asai et al., 2009) and measured 166, 164 and 159 segments at 65, 90 and 185 hours a.n.d., respectively (Fig. 4). At 65 hours a.n.d., the mean and standard deviation (SD) of the segment length distribution were 9.11 and 2.93 cells, respectively, increasing to 11.35 and 3.85 cells at 90 hours a.n.d. At 185 hours a.n.d., the mean and SD were 11.11 and 3.81 cells, respectively. Especially, the distribution at 65 hours a.n.d. peaked at approximately 8 cells, whereas those at later times peaked at 10 cells. In addition, the distribution of segment length was narrower at 
the early stage than at the later stage. The distributions at 65 and 90 hours a.n.d. were significantly different $\left(p=2.10 \times 10^{-4}\right.$; two-sample two-sided Kolmogorov-Smirnov test), whereas no significant difference appeared between the distributions at 90 and 185 hours a.n.d. ( $p=0.999$; same test). From the cell lineage analysis, we determined the average segment length after 69, 70, 71, 72 hours a.n.d. as approximately 12 cells. Especially, no significant difference was observed between the distributions at 69 and 90 hours a.n.d. ( $p>0.05$; student's $t$-test). These analyses suggest that, under our culture conditions, the heterocyst pattern formation reaches steady state at 69 hours a.n.d.

The heterocyst patterns observed at the early (63-65 hours a.n.d.) and later (69hours a.n.d.) stages were named "early pattern" and "later pattern", respectively. When estimating kinetic parameter values in our mathematical model, the segment length distributions at 65 and 90 hours a.n.d. were regarded as phenotypes of the early and later patterns, respectively.

\subsection{One dimensional Cellular Automaton model of heterocyst pattern formation}

We constructed a cellular automaton model (CA model) of the heterocyst pattern formation in nitrogen-deprived conditions, incorporating cell division and differentiation. The essential components of the model are (i) stochastic determination of cellular fate and (ii) cellular cooperation in a filament.

The CA model consists of a one-dimensional row of cells representing a filament. Individual cells are classified into vegetative cell or heterocyst. The dynamics of vegetative cell is modeled by a stochastic transition process involving cell division, differentiation into heterocyst and increase of cell age. The differentiation of a 
vegetative cell into a heterocyst is irreversible (Fig. 5). The cell age is a hypothetical discrete number, which stochastically increases over time in each vegetative cell. When a cell divides, the cell age is initialized to its minimum value (1). The cell age stochastically increases by one step up to its maximum value (10), or until the cell divides or differentiates. We call the increase of cell age "maturation", and the increasing rate of cell age "maturation rate", respectively. After a cell division, the original cell is replaced by two daughter cells with cell age of 1 . Following a cell division, each daughter cell independently follows a stochastic process of cell division, differentiation and maturation. Cells that have differentiated into heterocysts undergo no further dynamic changes.

Our assumed cell division and differentiation depend on the cell age. As the cell age increases, the cell will more likely to divide or differentiate.

In addition, the site of differentiation is also determined by lateral inhibition from the two existing heterocysts flanking the segment (Meeks and Elhai, 2002). Inter-segment cooperation exerts little or no effect on heterocyst location. Here, we determined the effect of lateral inhibition directly from an experimental result. Rivers reported that the lateral inhibition acts through paracrine signaling, which diffuses over several cell diameters from a heterocyst (Rivers et al., 2014). In intercellular signaling for the heterocyst pattern formation, a positive feedback regulation for het $R$ gene expression by the hetR translational product, HetR, plays a critical role (Buikema and Haselkorn, 1991; Black et al., 1993; Zhou et al., 1998). Thus, as the number of inhibitory molecules in the intermedium region of a segment decreases, the level of the HetR (activator) protein acutely and sharply increases. This is the reason why we used the sigmoidal function for the lateral inhibition with Hill coefficients, depending on how 
an individual cell is far from heterocysts. In this model, vegetative cells close to either heterocyst are less likely to differentiate than more distant cells.

The division rate $p(i)$, differentiation rate $q(i)$, maturation rate $r(i)$, and lateral inhibition rate $l(n)$ of the cells are modeled as follows. Our assumed cell division and differentiation rates are satisfied by sigmoidal Hill functions of the cell age $i$. As mentioned above, we similarly describe the lateral inhibition by a sigmoidal Hill function of $n$, which is the number of vegetative cells between a focal cell and each heterocyst in a segment.

$$
\begin{aligned}
& p(i)=a \cdot \frac{i^{m_{1}}}{i^{m_{1}}+k_{1}^{m_{1}}} \\
& q(i)=b \cdot \frac{i^{m_{2}}}{i^{m_{2}}+k_{2}^{m_{2}}} \quad \text { (1), Model 1 } \\
& r(i)=c \\
& l(n)=d \cdot \frac{n^{f}}{n^{f}+e^{f}},
\end{aligned}
$$

where the kinetic parameters $a(1 / t), b(1 / t)$, and $c(1 / t)$ denote the maximum division, differentiation and maturation rates, respectively, without lateral inhibition from heterocysts ( $t$ is the time step). $k_{1}$ and $k_{2}$ are the maturation levels of the cell age required for $50 \%$ activation of cell division and differentiation, respectively. The cooperativity of cell age activation is parameterized by the Hill coefficients $m_{1}$ and $m_{2}$, respectively. The rate of lateral inhibition depends on the kinetics parameters $d, e$ and $f$. The parameter $d$ represents the rate how the differentiation can be inhibited in the region far from a heterocyst (the lateral inhibition rate gets closer to the parameter $d$ when the 
value of $n$ is large enough). The parameter $e$ is the number of vegetative cells, which is between the focal cell and either flanking heterocyst, required for 50\% inhibition. In response to the number of vegetative cells from either heterocyst, the cell differentiates with a tendency determined by the parameter $f$.

To model the lateral inhibition from each of the terminal heterocysts, $l\left(n_{l}\right)$ and $l\left(n_{r}\right)$ are multiplied by the differentiation rate (here, $n_{l}$ and $n_{r}$ are the number of vegetative cells between the focal cell and the left and right flanking heterocysts, respectively). Additionally, a new heterocyst is assumed to immediately suppress the differentiation of neighboring cells. According to previous studies, compounds stored in cyanophycin are catabolized into early-stage fixed-nitrogen products, which are then released (Picossi et al., 2004). A differentiating cell also releases a diffusible protein that suppresses the differentiation of neighboring cells before it can sufficiently mature (Yoon and Golden, 1998, 2001). Therefore, immediate suppression is an appropriate simplification for the lateral inhibition system.

The kinetics parameters $m_{1}, m_{2}, k_{1}$, and $k_{2}$ in the cell division and differentiation rates of Model 1 were determined from the cell lineage as described below. The remaining kinetic parameters $a, b, c, d, e$ and $f$ are not directly estimable from the experimental data, and were instead estimated by mathematical analysis (see Section 2.5.).

The parameters $m_{1}$ and $k_{1}$ in the cell division rate $p(i)$ were decided from the experimental distribution of cell cycle length. Cellular growth and division were assumed independent of cells' positions in the filament (Asai et al., 2009). Given that 93.2\% of the cells divided within 13 hours in the generations 2-4 (Fig. 3C), we assumed cell cycle length below 13 hours in the analysis. First, we summed the number of cells 
of each cell cycle length in the generations 2, 3 and 4. Second, we normalized these results by the total number of cells (which had divided within 13 hours in the generations 2-4). Finally, we standardized the cell cycle length $\in[0,13]$ hours to the cell age $\in[1,10]$ a.u., and fitted a sigmoidal curve to the experimental data. The Hill coefficients obtained by regression analysis were $m_{1}=6.25$ and $k_{1}=6.99$ (Fig. 6A). For simplicity, we hereafter set $m_{1}=6$ and $k_{1}=7$ in the cell division rate $p(i)$.

The parameters $m_{2}$ and $k_{2}$ in the differentiation rate $q(i)$ were similarly obtained from the experimental data. However, the number of early-stage heterocysts in the cell lineage was not enough to accurately determine the differentiation rate. Thus, the differentiation rate was determined from later-stage heterocysts. By measuring the duration time length between the latest cell division and differentiation, we found that all heterocysts differentiated within 13 hours. This result indicates that the differentiation and cell division occur on the same timescale. As before, the time length $\in[0,13]$ hours were standardized to the cell age $\in[1,10]$ a.u., and fitted to the experimental data. The differentiation frequency was also a sigmoidal function of the cell age. The Hill coefficients obtained by regression analysis were $m_{2}=10.0$ and $k_{2}=7.55$ (Fig. 6B). For simplicity, we hereafter set $m_{2}=10$ and $k_{2}=8$ in the differentiation rate $q(i)$.

\subsection{Numerical Simulation}

The dynamics of heterocyst pattern formation was computed by Monte Carlo simulation. We applied a random sequential update for the time evolution, whereby the state transition of a randomly selected cell is examined at each time step. The time step 
$\Delta t$ was given by $\alpha \cdot r(i) / n$ (where $\alpha$ corresponds to the time required to increase the cell age of all cells by one step, and $n$ is the number of vegetative cells in a filament). From Eq. (1), the division, differentiation and maturation probabilities were determined as $p(i) \Delta t, q(i) \Delta t$ and $r(i) \Delta t$, respectively.

To calculate the heterocyst pattern formation under nitrogen deprived conditions, the initial configuration was a one-dimensional filament of vegetative cells flanked by two heterocysts. The number of vegetative cells in a filament and the cell age of each cell were randomly selected. Vegetative cells were allowed to stochastically divide, differentiate or mature until a filament acquired 5000 cells. If all vegetative cells differentiated into heterocysts before the filament had grown to 5000 cells, the result was discarded and a new filament was simulated and analyzed. Without changing the kinetic parameter values, we simulated and accumulated the heterocyst pattern formations in 100 filaments. In five separate runs of this simulation process, we observed no significant differences among the generated distributions $(p>0.95$, two-sample two-sided Kolmogorov-Smirnov test). Thus, we can generate the steady-state distribution of segment length in a single simulation process.

\subsection{An approximate two-step Markov analysis of heterocyst pattern formation}

Here we characterize the heterocyst pattern by the segment length, which is an alignment of vegetative cells enclosed by two heterocysts. We determined the steady-state distribution of segment length by Markov process approximations.

The analysis was conducted in two steps. In the first step, we determined the steady-state cell age distribution among the vegetative cell population. In the second step, we determined the steady-state distribution of segment length among the segment 
population.

First, we assumed that the transition process including the cell division, differentiation and maturation are independent among cells using a mean field approximation. The cell age dynamics then follows a Markov process. The differentiation rate in Model 1 is multiplied by the average rate of lateral inhibition, itself approximated by a step function. The inhibition rate of a cell located $e$ cells from either heterocyst in a segment is $50 \%$, except for the lateral inhibition coefficient $d$. The averaged lateral inhibition rate in a segment is then approximated by $d / 2 e\left(=l^{\prime}\right)$, including the lateral inhibition coefficient.

We derived the following master equation for the cell age transition process involving the cell division, differentiation and maturation:

$$
\begin{aligned}
& \frac{d}{d t} s(1)=-\left(p(1)+q(1) \cdot l^{\prime}+r(1)\right) \cdot s(1)+\sum_{i=1}^{i=10} 2 p(i) \cdot s(i) \\
& \frac{d}{d t} s(2)=r(1) \cdot s(1)-\left(p(2)+q(2) \cdot l^{\prime}+r(2)\right) \cdot s(2) \\
& \ldots \\
& \frac{d}{d t} s(9)=r(8) \cdot s(8)-\left(p(9)+q(9) \cdot l^{\prime}+r(9)\right) \cdot s(9) \\
& \frac{d}{d t} s(10)=r(9) \cdot s(9)-\left(p(10)+q(10) \cdot l^{\prime}\right) \cdot s(10)
\end{aligned}
$$

where $s(i)$ is the frequency of the cell age $=i$.

In our model, the long-term distribution of cell age is the eigenvector associated with the highest eigenvalue of the transition matrix. We confirmed that the 
analytically solved cell age distribution was consistent with that obtained by numerical simulation using the same kinetic parameter values.

Second, we assumed that each segment evolves independently of other segments. Here we assumed that the transition dynamics of each cell age is at the steady state. We can use the approximated division and differentiation rates which are averaged by the frequency of each cell age at the steady state. The dynamics of the segment length then follows a Markov process. Since longer segments contain more dividing cells, the growth speed of a segment is proportional to its length. Each cell that differentiates into a heterocyst divides the segment into two shorter segments (Fig. 7). The lateral inhibition effect is directly included by placing a heterocyst at both ends of the segment (Fig. 7).

From the steady-state distribution of cell age derived in the first step of the analysis, the average division rate $\hat{p}$ and differentiation rate $\hat{q}$ are given by

$$
\begin{aligned}
& \hat{p}=\sum_{i=1}^{i=10} s(i) \cdot p(i) \\
& \hat{q}=\sum_{i=1}^{i=10} s(i) \cdot q(i)
\end{aligned}
$$

We derived the following master equation for the segment length transition: 


$$
\begin{aligned}
& \frac{d}{d t}(x(1), x(2), x(3), \cdots, x(n-1), x(n))^{\mathrm{T}} \\
& =\left(\begin{array}{cccccc}
X_{1} & 2 \cdot l(1) \cdot l(2) \cdot \hat{q} & 2 \cdot l(2) \cdot l(2) \cdot \hat{q} & \cdots & 2 \cdot l(n-2) \cdot l(2) \cdot \hat{q} & 2 \cdot l(n-1) \cdot l(2) \cdot \hat{q} \\
\hat{p} & X_{2} & 2 \cdot l(1) \cdot l(3) \cdot \hat{q} & \cdots & 2 \cdot l(n-3) \cdot l(3) \cdot \hat{q} & 2 \cdot l(n-2) \cdot l(3) \cdot \hat{q} \\
0 & 2 \hat{p} & X_{3} & \cdots & 2 \cdot l(n-4) \cdot l(4) \cdot \hat{q} & 2 \cdot l(n-3) \cdot l(4) \cdot \hat{q} \\
\vdots & \vdots & \vdots & \vdots & \vdots & \vdots \\
0 & 0 & 0 & \cdots & X_{n-1} & 2 \cdot l(1) \cdot(n) \cdot \hat{q} \\
0 & 0 & 0 & \cdots & (n-1) \hat{p} & X_{n}
\end{array}\right)\left(\begin{array}{c}
x(2) \\
x(3) \\
\vdots \\
x(n-1) \\
x(n)
\end{array}\right) \\
& X_{i}=-i \cdot \hat{p}-\sum_{j=1}^{j=i} l(j) \cdot l(i+1-j) \cdot \hat{q},
\end{aligned}
$$

where $x(n)$ is the frequency of the segment length $n$. In the analytical solution, we set maximum $n=50$.

Similar to the long-term cell age distribution, we assumed the long-term distribution of segment length as the eigenvector associated with the largest eigenvalue of the transition matrix. We confirmed agreement between the analytical solution and numerical distribution of segment length over a wide range of kinetic parameter values (some examples are shown in Fig. 8). Therefore, the approximate two-step Markov analysis properly explains the steady-state distribution of segment length. Hereafter, the steady-state distribution of segment length was analytically calculated.

\subsection{Estimation of the kinetic parameter values describing the internal dynamics of heterocyst pattern formation}

To estimate the kinetic parameter values for the later pattern formation, we compared the analytical solution with the experimental distribution of segment length. 
We searched for kinetic parameter values that best explained the experimental data by a Monte Carlo method.

First, we randomly selected kinetic parameter values from specified regions $(a \in[0.01,0.1], b \in[0.1,1.0], c \in[0.05,0.95], d \in[0.5,1.6], e \in[5,30], f \in[3,10])$. We then calculated the analytical solution, and the Kullback-Leibler divergence ( $\mathrm{K}-\mathrm{L}$ divergence) between the analytical solution and the experimental distribution. Values of $a$ were randomly selected at 0.01 intervals, those of $b, c$ and $d$ at 0.1 intervals, and those of $e$ and $f$ at intervals of 1 . Implementing this procedure on $5 \times 10^{6}$ kinetic parameter sets, we selected kinetic parameter values that minimized the $\mathrm{K}-\mathrm{L}$ divergence.

The kinetic parameter values most consistent with the experimental distribution $(\mathrm{K}-\mathrm{L}$ divergence $=0.0358)$ were $(a, b, c, d, e, f)=(0.06,0.7,0.25,0.8,10,6)$. We confirmed that, by inserting the optimal parameter values into the analytical solution, we faithfully reproduced the later pattern of the experimental data (Fig. 9).

To investigate the sensitivity of later pattern formation to the kinetic parameters, we separately varied each parameter and determined the parameter limits for which the $\mathrm{K}-\mathrm{L}$ divergence remained small. Since the minimum $\mathrm{K}-\mathrm{L}$ divergence approximates the maximum logarithmic likelihood, the limits can be determined in the context of Bayesian inference. Taking a uniform prior probability, the posterior probability density function $\operatorname{Pr}(\theta \mid$ data $)(\theta=\{a, b, c, d, e, f\})$ is given by $\operatorname{Pr}($ data $\mid \theta) / \int \operatorname{Pr}($ data $\mid \theta) d \theta$. The data in the probability density function are obtained by Markov Chain Monte Carlo (MCMC) sampling.

As shown in Fig. 10, the kinetic parameter values that maximize the posterior probability are those that minimize the $\mathrm{K}-\mathrm{L}$ divergence. The parameter limits are determined within the $90 \%$ confidence interval, constructed such that the posterior 
probabilities within the interval always exceed those outside the interval. For each parameter, the limits were computed while other parameters were fixed at estimated values. In this way, the limits were determined as $a \in[0.05,0.07], b \in[0.6,0.8]$, $c \in[0.15,0.75], d \in[0.7,0.9], e=10, f \in[5,7]$. Therefore, the later pattern formation is relatively robust to the maturation rate $c$, and is most sensitive to the value of $e$, the number of vegetative cells between a focal cell and existing heterocysts. Previous experimental studies have suggested that the placements of new heterocysts along the filament are largely governed by the diffusivity of PatS (Risser and Callahan, 2009). Our theoretical findings strongly corroborate this proposal.

\subsection{A model of the early pattern formation}

\subsubsection{Explanation of the early pattern formation using Model 1}

As mentioned above, the segment length distributions differed between the early and later stages of pattern formation. In particular, short segments were more common in the early than in the later patterns. Experimental studies have confirmed the release of nitrogen compounds and PatS from new heterocysts (Yoon and Golden, 1998, 2001), suggesting that lateral inhibition by heterocysts exerts the same effect at the early and later stages. We considered two alteration processes for the division, differentiation and maturation rates: i) only the kinetic parameter values vary using the same functional forms as Model 1, ii) both the kinetic parameter values and functional forms vary.

Initially, we adopted Model 1 for analyzing the early pattern formation. We assumed different kinetic parameter values between the early and later stages, but the same functional form. The kinetic parameter values for the early pattern $(a, b, c)$ were estimated by comparing the $\mathrm{K}-\mathrm{L}$ divergences between the analytical solution and 
experimental distribution of segment length. The analytical solution that best matched the experimental distribution $(\mathrm{K}-\mathrm{L}$ divergence $=0.0174)$ was obtained for $(a, b, c)=$ $(0.01,0.6,0.75)$.

However, the cell age distribution obtained from the estimated kinetic parameter values differed from the experimental results (Fig. 11). In the model distribution, more than $70 \%$ of the cells reached the maximum cell age (10), because the estimated maturation rate was much higher than the estimated division and differentiation rates. This implies that the cell division and differentiation do not occur when the cell age is smaller, and they occur only when the cell age reaches the maximum value 10 in the model. By contrast, cell division and differentiation occur frequently when the cell is young in experimental observations (Fig. 3C).

From these contradictory results, we inferred that Model 1 cannot fully explain the dynamics of early pattern formation. Therefore, we explored the feasibility of revising each functional form in the model.

Among the three transition rates in Model 1, the functional form of division rate requires no alteration between the early and later stages. The first heterocyst differentiation occurred much later than the timing when the disturbance of nitrogen depletion had disappeared in the cell cycle dynamics. Thus, we focused on the functional form of the differentiation rate; in particular, the dependency of differentiation on the cell age.

\subsubsection{Analysis of the activation of differentiation activating (hetR) gene transcription}

We previously reported that after cell division of the cells on the way of 
differentiation, the activity of $h e t R$ gene transcription decreases and is reset to the basal level of vegetative cells at the later stage (Asai et al., 2009). For this reason, we assumed that the differentiation rate is an increasing function of the cell age in Model 1.

We analyzed the activity of hetR gene transcription after cell division at the early and later stages by calculating the ratio of hetR::gfp fluorescence signal before or after cell division to that at 1 hour before cell division (Fig. 12, see Appendix G for calculation procedure). At 69 and 70 hours a.n.d., the fluorescence signal ratios at 0 hour after cell division were $0.966 \pm 1.71 \times 10^{-2}$ and $0.961 \pm 1.31 \times 10^{-3}$ (average $\pm S D$ ), respectively. The values were statistically different from those at 1 hour before cell division, respectively (student's $t$-test, $p<0.01$ ), suggesting that the activity of het $R$ gene transcription decreases after cell division during the later stage. Note that the activity did not decrease for examined mother cells before the cell division. Conversely, at 63, 64 and 65 hours a.n.d., the fluorescence signal ratios at 0 hour after cell division were $0.999 \pm 1.68 \times 10^{-2}, 1.003 \pm 1.80 \times 10^{-2}$, and $1.004 \pm 7.75 \times 10^{-3}$, respectively (average \pm SD). They are not statistically different from those at 1 hour before cell division, respectively (student's $t$-test, $p>0.01$ ). In addition, during the early stage, we did not observe significant differences of the ratio after the early-stage cell division, respectively (student's $t$-test, $p>0.01$ ). These results suggest that het $R$ gene transcription is not immediately perturbed by cell division but remains active at the early stage, thereby the activity of hetR gene transcription is considered to be independent of the cell age.

\subsubsection{Explanation of the early pattern formation using a new mathematical model}

Based on these findings, we proposed an alternative model of the early pattern 
formation, in which the differentiation is independent of the cell age:

$$
\begin{array}{ll}
p(i)=a \cdot \frac{i^{6}}{i^{6}+7^{6}} & \\
q(i) & =b \\
r(i) & =c
\end{array}
$$

Here, $p(i), q(i)$ and $r(i)$ are the cell division, differentiation and maturation rates, respectively.

The analytical solution was derived by an approximate two-step Markov analysis. As before, we estimated the optimal kinetic parameter values $(a, b, c)$ by comparing the $\mathrm{K}-\mathrm{L}$ divergences between the analytical solution and experimental distribution. The estimated optimal parameter values $(\mathrm{K}-\mathrm{L}$ divergence $=0.0136)$ were $(a, b, c)=(0.03,0.7,0.25)$, similar to those of the later pattern. Therefore, inserting the optimal parameter values of the later pattern $(a, b, c, d, e, f)=(0.06,0.7,0.25,0.8,10$, 6) into the analytical solution should also reproduce the experimental distribution (indeed, the $\mathrm{K}-\mathrm{L}$ divergence was 0.0238 ).

To investigate the sensitivity of early pattern formation to the kinetic parameters $(a, b, c)$, we again determined the limits in the context of Bayesian inference, obtaining $a \in[0.02,0.06], b \in[0.6,0.9], c \in[0.15,0.25]$. As shown in Fig. 13A, the kinetic parameter values that maximize the posterior probability also minimize the $\mathrm{K}-\mathrm{L}$ divergence ( $a=0.03, b=0.7, c=0.25$ ). The early pattern formation is more robust to wide changes in the maximum division rate $a$ than the later pattern formation. Given that the estimated values of $b$ and $c$ are the same at both stages, we discuss whether the 
estimated values of the remaining parameter $a(0.03$ and 0.06 at the early and later stages, respectively) are statistically different. The posterior probability of $a=0.06$ is included in the $90 \%$ confidence interval of $a=0.03$ (where the posterior probabilities within the interval always exceed those outside the interval). This result confirms our expectation that setting $a=0.06$ in Model 2 yields the correct dynamics of early pattern formation.

We then confirmed that the distribution of segment length in the early pattern is reproduced by the kinetic parameter values adopted in the later pattern, assuming that the differentiation is independent of the cell age (Fig. 14). The cell age distribution, obtained from the estimated kinetic parameter values, shows that young cells were more frequent than fully matured cells (Fig. 11). This implies that the cell division and differentiation frequently occurred before the cells reached the cell age 10 . We inferred that the differentiation is probably independent of the cell age during the early stage.

\subsubsection{Validation of the estimated parameter values to explain the early and later pattern formations}

Finally, we evaluated the validity to explain the early and later pattern formations with the same parameter $a=0.06$ using Model 1 and 2. We summed the logarithmic likelihoods of both models within the kinetic parameter range $a \in[0.01,0.1]$ (while fixing $(b, c, d, e, f)=(0.7,0.25,0.8,10,6))$, and calculated the posterior probability of each value of $a$. Logarithmic likelihoods generally depend on the data size. However, since the total number of segments was essentially the same at the early and late stages (164 and 166, respectively), we considered that the logarithmic likelihoods of each model equally contributed to their summations. 
Setting $a=0.06$, we found that the posterior probability was maximized (Fig. 13B). Therefore, we concluded that the same kinetic parameter values can explain the dynamics of both early and later pattern formations using Model 1 and 2.

\section{Discussion}

Combining experimental data and mathematical methods, this study investigated the dynamics of cell division and differentiation of the filamentous cyanobacterium, Anabaena sp. We analyzed cell lineage data obtained from the time-lapse images analysis, and found that the differentiation rates differ between the early and later stages of pattern formation. We then developed a mathematical method to describe these dynamics. We also developed a two-step Markov approximation to obtain the distribution of segment length. Using this approximation, we estimated the kinetic parameter values, including the differentiation rate, from the experimental data. The model that appropriately described the later-stage dynamics proved inadequate for early-stage dynamics, even when the kinetic parameters were tuned. We needed to consider different functional form of differentiation rate. It indicates that the mechanisms of differentiation differ between the early and later stages.

In our previous study, we found that the frequency of cells expressing the hetR gene was higher at early than later stages (Asai et al., 2009). We newly revealed quantifiable differences of the heterocyst patterns between the both stages. Especially, the early pattern was characterized by short segment length, as another group reported (Yoon and Golden, 2001). It has been considered that metabolic functions or cellular cooperation alters during the heterocyst pattern formation (Borthakur et al., 2005; Allard et al., 2007). However, the extent to which these changes in single cells affect the 
heterocyst pattern formation has not been quantitatively discussed.

To quantitatively understand the intercellular dynamics at the early and later stages, we analyzed the cell lineage obtained from the time-lapse images, again. At the later stage, differentiation depended on the time elapsed after the latest cell division. We previously reported that, if differentiating cells divided during the later stage, the activity of hetR gene transcription decreased and the differentiation seemed to be cancelled (Asai et al., 2009). This is another reason that we consider the differentiation rate depends on the cellular maturation.

In our proposed mathematical model, cell division and differentiation depend on the cellular maturation, "cell age". We demonstrated the dynamics of our model by numerical simulation and by an approximate two-step Markov analysis. In applying the Markov approximations to the cell age and segment length transition dynamics, we made the following assumptions:

i) Cells autonomously and independently carry out the transition processes of cell division, differentiation and maturation,

ii) The transition process of each cell age is at the steady state.

In the experimental observations, we revealed that the cell cycle and cell division processes are asynchronous among the cultured cells, which supports the assumption i) (Asai et al., 2009). Here, we revealed that the differentiation initiates after the disturbance of cell cycle due to nitrogen depletion had disappeared. In addition, we considered that the temporal pattern of differentiation at the steady state differs between cells at the early stage and those at the later stage. Therefore, we interpret that the dynamics of cell age transition is at the steady state at each stage. Then, mathematical assumption ii) is evaluated to be valid biologically. Under the assumptions i) and ii), the 
frequency of segment length transition is at the steady state. We can use this mathematical method to understand the heterocyst pattern formation at various stages.

We estimated the most optimal kinetic parameter values by comparing the analytical solution with the experimental distribution. Here, we discuss the biological validity of the optimal kinetic parameters in our model. When the expressions of PatS and another negative regulator of the differentiation, HetN, are suppressed, all vegetative cells differentiate into heterocysts under nitrogen deprived conditions (Borthakur et al., 2005). This indicates that many more cells can potentially differentiate than normally observed. In our mathematical model, almost all vegetative cells differentiate into heterocysts without the lateral inhibition (that is, $l(n)=1$ ) when the estimated maximum division rate $a$ and differentiation rate $b$ were inserted. It suggests the biological validity of estimated kinetic parameter values. We consider that the differentiation capability is usually suppressed by lateral inhibition from nearby heterocysts.

Analyzing hetR gene transcription in single cells, we inferred that differentiation occurs independently of cellular maturation during the early stage. Toyoshima and his colleagues also measured the PhetR::gfp signal from nitrogen-deprived cells of the same strain used in our work (Toyoshima et al., 2009). They reported that the het $R$ gene is simultaneously transcribed in all vegetative cells at early time of heterocyst pattern formation. Bradley and Carr reported that all vegetative cells begin phycobilisome degradation prior to the first heterocyst appearance in nitrogen depleted environments (Bradley and Carr, 1976). These studies suggest that vegetative cells at any stage of maturation are primed for differentiation at the start of heterocyst pattern formation. 
Here, we considered the reason why the dynamics of hetR gene expression changes during the pattern formation. It appears that intercellular nitrogen depletion triggers a rapid $\mathrm{Ca}^{2+}$ increase that activates hetR gene transcription via the transcriptional factors NrrA and NtcA, regardless of cellular maturation (Herrero et al., 2004; Ehira and Ohmori, 2006). Then, the hetR gene expression is activated rapidly. On the other hands, at the later stage, $\mathrm{Ca}^{2+}$ does not accumulate so much and the expression of transcriptional factors increase gradually after cell division (Muro-Pastor et al., 2001; Muro-Pastor et al., 2002; Li et al., 2003; Ehira and Ohmori, 2006). Then, the hetR gene expression is activated gradually. In this way, the molecular network to activate the het $R$ gene expression is basically different at the early and later stages. Especially, at the early stage, the amount of HetR protein is relatively larger and beyond the amount of the lateral inhibition factors in a cell. Therefore, the response to the lateral inhibition is unreceptive irrespective of the cell age. As the pattern formation proceeds, only matured cells get unreceptive to the lateral inhibition factors, thereby they differentiate into heterocyst.

We conclude that the early and later patterns differ because of different dependencies of differentiation on the cell age. Under this assumption, our model results are consistent with experimental distributions at both stages, without changing the kinetic parameter values. Although the dynamics of heterocyst pattern formation have been frequently reported, and the distinction between early and later stages is well-known, our approach has enabled the first theoretical understanding of the phenotypic changes occurring during heterocyst pattern formation. We consider that the transition in differentiation processes as an adaptive system against environmental changes. To rapidly respond to a nitrogen depleted environment, Anabaena filaments 
must increase the number of their heterocysts. Consequently, differentiation is favored over cell division and maturation at the early stage. However, both differentiation and nitrogen fixation processes are costly to consume lot of energy source. Thus, the number of heterocysts could be more properly regulated for optimal growth at the later stage. We model this two-phasic differentiation processes not by changing the kinetic parameter values but by assuming a different dependency on cellular maturation. Our findings, together with the approximate two-step Markov analysis, provide novel approaches for capturing the dynamics of heterocyst pattern formation at various stages.

\section{Acknowledgements}

We express our sincere thanks to Dr. Bernold Fiedler (Freie Universität Berlin) for his comments to our data analysis. We also thank members of the Mochizuki Lab (RIKEN), Hironori Asai, Shunsuke Iwamori and members of Iwasaki Lab (Waseda University) for their valuable comments and advice. This study was supported in part by Grants-in-Aid from JST CREST program of Japan (to A.M.), and the Japanese Society for the Promotion of Science 11J06592 (to J.I.), 23687002 and 00324393 (to H.I.). 


\section{Appendix A. Culture system}

Anabaena sp. PCC 7120 and its derivative PhetR::gfp reporter strain (SRhetR-1Gn strain; Asai et al., 2009), which harbors a transcriptional fusion of a promoterless $g f p$ gene and the het $R$ gene promoter, were grown in BG- 11 or BG- $11_{0}$ (lacking sodium nitrate) liquid medium at $30{ }^{\circ} \mathrm{C}$ under continuous illumination by a fluorescent lamp at $45 \mu \mathrm{M}$ photons $\mathrm{m}^{-2} \mathrm{~s}^{-1}$ as described in (Rippka et al., 1979). The liquid culture was bubbled with air (normal $\mathrm{CO}_{2}$ ). For heterocyst induction on plates, Anabaena was grown in liquid media to an approximate optical density at $730 \mathrm{~nm}$ $\left(\mathrm{OD}_{730}\right)$ of 0.2 , washed four times with $\mathrm{BG} 11_{0}$ liquid medium, diluted to an $\mathrm{OD}_{730}$ of $\sim 0.01$, and spread onto a BG11 $1_{0}$ solid $(1.5 \%$ agar $)$ medium. Filaments of the SRhetR-1Gn strain on $\mathrm{BG} 11_{0}$ solid media were grown at $30{ }^{\circ} \mathrm{C}$ under continuous fluorescent lamp illumination (FL30SW-B, $50 \mu \mathrm{Em}^{-2} \mathrm{~s}^{-1}$; Hitachi Co., Tokyo, Japan).

\section{Appendix B. Monitoring system}

Filaments of the SRhetR-1Gn strain on BG- $11_{0}$ solid media were observed with an Olympus IX-71 inverted microscope (20x objective LUCPlanFLN lens, NA 0.45, Olympus, Tokyo, Japan) at $30{ }^{\circ} \mathrm{C}$. Exchange of lamps, mirror units, and sample positions were programmed with the Slidebook 4.1 software (Intelligent Imaging Innovations, Denver, CO, USA). For bright field and fluorescence microscopic observations, we used a chilled charge-coupled device (CCD) camera (PIXIS1024, Princeton Instruments, Trenton, NJ, USA), also controlled by Slidebook 4.1 software. During heterocyst differentiation, green fluorescent protein (GFP) and phycobilisome fluorescence were monitored using filter sets U-MNIBA3 (Olympus) and U-MWIG3 (Olympus), respectively. 


\section{Appendix C. Statistical analysis}

The statistical equalities between the two data samples were evaluated by two-sample Kolmogorov-Smirnov tests. Quantities evaluated were the distributions of duration time after cell division, the distributions of cell cycle length in the generations $1-4$, and the experimental and analytical distributions of segment length. First, we made a cumulative frequency distribution for each sample, using the same intervals for both distributions. For each interval, we subtracted one step function from the other function. $S_{n 1}(X)$ and $S_{n 2}(X)$ are the cumulative step functions of each sample, respectively. The Kolmogorov-Smirnov test focuses on

$$
D=\operatorname{maximum}\left|S_{n 1}(X)-S_{n 2}(X)\right|
$$

where $D$ is the maximum deviation statistic between two samples.

To calculate the $p$-values, it has been shown that

$$
K=4 D^{2} \frac{n_{1} n_{2}}{n_{1}+n_{2}}
$$

obeys a $\chi^{2}$ distribution with two degrees-of-freedom at both sides when both sample sizes $n_{1}$ and $n_{2}$ exceed 40 (Siegel, 1956).

\section{Appendix D. Numerical experiments}

The analytical solutions were computed by a program written in the $\mathrm{C}$ programming language and Mathematica 6 software (Wolfram). Programs for the 
additional numerical experiments, including the Cellular Automaton simulation, were also constructed in $\mathrm{C}$ programing language.

\section{Appendix E. Calculation of the Kullback-Leibler divergence}

To estimate the kinetic parameter values, we compared the analytical solution with the experimental distribution of segment length. The Kullback-Leibler divergence $I(g ; f)$ was calculated as

$$
\begin{aligned}
I(g ; f) & =\sum_{i=1}^{i=m} g(i) \cdot \log \frac{g(i)}{f(i \mid \theta)} \\
& =\sum_{i=1}^{i=m} \frac{n_{i}}{N} \cdot \log \left(\frac{n_{i}}{N}\right)-\sum_{i=1}^{i=m} \frac{n_{i}}{N} \cdot \log f(i \mid \theta) \quad\left(\because g(i)=\frac{n_{i}}{N}, N=\sum_{i=1}^{i=m} n_{i}\right),
\end{aligned}
$$

where $g(i)$ is the frequency of segment length $i$ in the experimental distribution, and $f(i \mid \theta)$ is the analytical solution given the kinetic parameter set $\theta=(a, b, c, d, e, f) . n_{i}$ is the number of segments of length $i$, and $N$ is the total number of segments in the experimental data. The maximum number of segment length $(m=50)$ was the maximum number yielded by the analytical solution.

The second term in the right-hand side of this expression denotes the averaged logarithmic likelihood $\mathrm{E}_{\mathrm{G}}[\log f(X \mid \theta)]$, from which we calculated the logarithmic likelihood $L(\theta)$ as $\sum_{i=1}^{i=m} n_{i} \cdot \log f(i \mid \theta) \quad\left(=N \cdot \mathrm{E}_{\mathrm{G}}[\log f(X \mid \theta)]\right)$. As mentioned previously, the minimum value of the $\mathrm{K}$ - $\mathrm{L}$ divergence corresponds to the maximum value of the (averaged) logarithmic likelihood. 


\section{Appendix F. Markov Chain Monte Carlo (MCMC) sampling for determining the posterior probability}

Having estimated the kinetic parameter values, we calculated the posterior probabilities of all parameter values. The posterior probability density function $\operatorname{Pr}(\theta \mid$ data $)$ obeys the following equation based on Bayes' theorem

$$
\operatorname{Pr}(\theta \mid \text { data })=\frac{\operatorname{Pr}(\text { data } \mid \theta) \cdot \operatorname{Pr}(\theta)}{\int \operatorname{Pr}(\text { data } \mid \theta) \cdot \operatorname{Pr}(\theta) d \theta} \propto \operatorname{Pr}(\text { data } \mid \theta) \cdot \operatorname{Pr}(\theta)
$$

where $\operatorname{Pr}(\theta)$ denotes the prior probability density function, and $\int \operatorname{Pr}($ data $\mid \theta) \cdot \operatorname{Pr}(\theta) d \theta$ denotes the normalization constant. We assumed that the prior distribution of $\operatorname{Pr}(\theta)$ is constant. Data samples were generated by the Metropolis algorithm as follows. We first selected the value of $a$ from the range $a \in[0.01,0.1]$, fixing the remaining kinetic parameters at their estimated values. We then randomly selected $a+0.01$ or $a-$ 0.01 (denoting this parameter as $\left.a^{\text {new }}\right)$. If $L\left(a^{\text {new }}\right)$ exceeded $L(a), a$ was replaced by $a^{\text {new }}$; conversely, if $L\left(a^{\text {new }}\right)$ was smaller than $L(a), a$ was modified to $a^{\text {new }}$ with probability $\exp \left(L\left(a^{\text {new }}\right)-L(a)\right)$. This process was conducted for $1 \times 10^{4}$ changes of the initial kinetic parameter value.

The $\operatorname{Pr}(\theta \mid$ data $)$ of the remaining kinetic parameters were computed in the same manner. Kinetic parameter values were selected from $b \in[0.1,1], c \in[0.05,0.95]$, $d \in[0.5,1.6], e \in[5,30]$, and $f \in[3,10]$. The kinetic parameters $b, c$, and $d$ were varied by 0.1 intervals, whereas $e$ and $f$ were varied by intervals of 1 . 


\section{Appendix G. GFP fluorescence analysis}

We analyzed the time-lapse images of the PhetR::gfp strain grown under the microscope in nitrogen-deprived culture by ImageJ software (NIH). The same images were used for the Movie 2A in our previous report (Asai et al., 2009). We traced each cellular boundary and measured the mean intensity of GFP fluorescence per area ("FL") and its background signal near each cell (“ $B G$ "). The subtracted fluorescence intensity was considered as relative hetR::gfp fluorescence signal (“FS"). Here, we referred to a lineage of mother and two sibling cells, which divided from the same mother cell, as "sample line" (Fig. G1). The number of sample lines $N$ is equal to the number of cells, which divided at 63, 64, 65, 69 and 70 hours a.n.d., respectively ( $N$ is 21 at 63 hours, 14 at 64 hours, 12 at 65 hours, 18 at 69 hours, and 14 at 70 hours a.n.d.). The fluorescence signal of mother cell at $i$-hour before cell division is represented as " $F S_{m-i, j}$ " (j: sample line number, $j \in[1, N]$ at each hour a.n.d.). On the other hands, the fluorescence signals of two sibling cells at $i$-hour after cell division are represented as " $F S_{s 1-i, j}$ " and " $F S_{s 2-i, j} "$, respectively.

Initially, we calculated the ratio of fluorescence signal before or after cell division to that at 1 hour before cell division in the same sample line as follows.

$$
\begin{aligned}
& r_{m-i, j}=F S_{m-i, j} / F S_{m-1, j} \\
& r_{s-i, j}=F S_{s-i, j} / F S_{m-1, j} \quad(s \text { denotes } s 1 \text { or } s 2) \quad(\mathrm{G} 1)
\end{aligned}
$$

Then, we averaged the fluorescence signal ratio at the same hour before or after cell division among different sample lines in which cell division occurred at 63, 64, 65, 
69, 70 hours a.n.d., respectively.

$$
\begin{aligned}
& r_{m-i}=\frac{1}{N}\left(\sum_{j=1}^{j=N} r_{m-i, j}\right) \\
& r_{s-i}=\frac{1}{2 N}\left(\sum_{j=1}^{j=N} r_{s 1-i, j}+\sum_{j=1}^{j=N} r_{s 2-i, j}\right)
\end{aligned}
$$

In Fig. 12, we show the average fluorescence signal ratio with standard deviation. 


\section{References}

Allard, J.F., Hill, A.L., Rutenberg AD (2007) Heterocyst patterns without patterning proteins in cyanobacterial filaments. Dev Biol 312: 427-434.

Asai, H., Iwamori, K., Kawai, S., Ehira, S., Ishihara, J., Aihara, K., Shouji, S., Iwasaki, H., 2009. Cyanobacterial cell lineage analysis of the spatiotemporal hetR expression profile during heterocyst pattern formation in Anabaena sp. PCC 7120. PLoS ONE 4(10), e7371.

Black, T.A., Cai, Y., Wolk, C.P., 1993. Spatial expression and autoregulation of hetR, a gene involved in the control of heterocyst development in Anabaena. Mol. Microbiol. 9, 77-84.

Borthakur, P.B., Orozco, C.C., Young-Robbins, S.S., Haselkorn, R., Callahan, S.M., 2005. Inactivation of patS and hetN causes lethal levels of heterocyst differentiation in the filamentous cyanobacterium Anabaena sp. PCC 7120. Mol. Microbiol. 57, 111-123.

Bradley, S., Carr, N.G., 1976. Heterocyst and nitrogenase development in Anabaena cylindrica. J. Gen. Microbiol. 96, 175-184.

Buikema, W.J., Haselkorn, R., 1991. Characterization of a gene controlling heterocyst differentiation in the cyanobacterium Anabaena 7120. Genes Dev. 5, 321-330.

Callahan, S.M., Buikema, W.J., 2001. The role of HetN in maintenance of the heterocyst pattern in Anabaena sp. PCC 7120. Mol. Microbiol. 40, 941-950.

Cumino, A.C., Marcozzi, C., Barreiro, R., Salerno, G.L., 2007. Carbon cycling in Anabaena sp. PCC 7120 sucrose synthesis in the heterocysts and possible role in nitrogen fixation. Plant Physiol. 143, 1385-1397. 
Ehira, S., Ohmori, M., 2006. NrrA directly regulates expression of hetR during heterocyst diffrentiation in the Cyanobacterium Anabaena sp. PCC 7120. J. Bacteriol. 188, 8520-8525.

Flores, E., Herrero, A., Wolk, C.P., Maldener, I., 2006. Is the periplasm continuous in filamentous multicellular cyanobacteria? TRENDS in Microbiol. 14, 439-443.

Golden, J.W., Yoon, H.S., 2003. Heterocyst development in Anabaena. Curr. Opin. Microbiol. 6, 557-563.

Herrero, A., Muro-Pastor, A.M., Valladares, A., Flores, E., 2004. Cellular differentiation and the NtcA transcription factor in filamentous cyanobacteria. FEMS Microbiol. Rev. 28, 469-487.

Huang, X., Dong, Y., Zhao, J., 2004. HetR homodimer is a DNA-binding protein required for heterocyst differentiation, and the DNA-binding activity is inhibited by PatS. Proc. Natl. Acad. Sci. U S A 101, 4848-4853.

Khudyakov, I.Y., Golden, J.W., 2004. Different functions of HetR, a master regulator of heterocyst differentiation in Anabaena sp. PCC 7120, can be separated by mutation. Proc. Natl. Acad. Sci. U S A 101, 16040-16045.

Kumar, K., Mella-Herrera, R.A., Golden, J.W., 2009. Cyanobacterial Heterocysts. Cold Spring Harbor Perspectives in Biology 2, a000315-334.

Li, J.H., Laurent, S., Konde, V., Bédu, S., Zhang, C.C., 2003. An increase in the level of 2-oxoglutarate promotes heterocyst development in the cyanobacterium Anabaena sp. PCC 7120. Microbiology 149, 3257-3263.

Mariscal, V., Herrero, A., Flores, E., 2007. Continuous periplasm in a filamentous, heterocyt forming cyanobacterium. Mol. Microbiol. 65, 1139-1145.

Meeks, J.C., Elhai, J., 2002. Regulation of cellular differentiation in filamentous 
cyanobacteria in free-living and plant-associated symbiotic growth states. Microbiol. Mol. Biol. Rev. 66, 94-121.

Muro-Pastor, M.I., Reyes, J.C., Florencio, F.J., 2001. Cyanobacteria perceive nitrogen status by sensing intracellular $\alpha$-ketoglutarate levels. J. Biol. Chem. 276, $38320-38328$.

Muro-Pastor, A.M., Valladares, A., Flores, E., Herrero, A., 2002. Mutual dependence of the expression of the cell differentiation regulatory protein HetR and the global nitrogen regulator NtcA during heterocyst development. Mol. Microbiol. 44, 1377-1385.

Picossi, S., Valladares, A., Flores, E., Herrero, A., 2004. Nitrogen-related genes for the metabolism of cyanophycin, a bacterial nitrogen reserve polymer. J. Biol. Chem. $279,11582-11592$.

Rippka, R., Deruelles, J., Waterbury, J.B., Herdman, M., Stanier, R.Y., 1979. Genetic assignments, strain histories and properties of pure cultures of cyanobacteria. J. Gen. Microbiol. 111, 1-61.

Risser, D.D., Callahan, S.M., 2009. Genetic and cytological evidence that heterocyst patterning is regulated by inhibitor gradients that promote activator decay. Proc. Natl. Acad. Sci. U S A 106, 19884-19888.

Rivers, O.S., Videau, P., Callahan, S.M., 2014. Mutation of sepJ reduces the intercellular signal range of a het $N$-dependent paracrine signal, but not of a patS-dependent signal, in the filamentous cyanobacterium Anabaena sp. strain PCC 7120. Mol. Microbiol. 94, 1260-1271.

Sakr, S., Jeanjean, R., Zhang, C.C., Arcondeguy, T., 2006. Inhibition of cell division suppresses heterocyst development in Anabaena sp. PCC 7120. J. Bacteriol. 188, 
1396-1404.

Sakr, S., Thyssen, M., Denis, M., Zhang, C.C., 2006. Relationship among several key cell cycle events in the development in Anabaena sp. PCC 7120. J. Bacteriol. 188, $5958-5965$.

Siegel, S., 1956. Nonparametric Statistics for the Behavioral Sciences, McGraw- Hill Book Company, New York, pp. 127-136.

Toyoshima, M., Sasaki, N., Fujiwara, M., Ehira, S., Ohomri, M., 2009. Early candidency for differentiation into heterocysts in the filamentous cyanobacterium Anabaena sp. PCC 7120. Arch. Microbiol. 192, 23-31.

Walsby, A.E., 2007. Cyanobacterial heterocysts: terminal pores proposed as sites of gas exchange. TRENDS in Microbiol. 15, 340-349.

Wilcox, M., Mitchison, G.J., Smith, R.J., 1973a. Pattern formation in the blue-green alga Anabaena. I. Basic mechanisms. J. Cell Sci. 12, 707-723.

Wilcox, M., Mitchison, G.J., Smith, R.J., 1973b. Pattern formation in the blue-green alga Anabaena. II. Controlled proheterocyst regression. J. Cell Sci. 13, 637-649.

Wolk, C.P., Quine, M.P., 1975. Formation of one-dimensional patterns by stochastic processes and by filamentous blue-green algae. Dev. Biol. 46, 370-382.

Wu, X., Liu, D., Lee, M.H., Golden, J.W., 2004. patS minigenes inhibit heterocyst development of Anabaena sp. strain PCC 7120. J. Bacteriol. 186, 6422-6429.

Yoon, H.S., Golden, J.W., 1998. Heterocyst pattern formation controlled by a diffusible peptide. Science $282,935-938$.

Yoon, H.S., Golden, J.W., 2001. PatS and products of nitrogen fixation control heterocyst pattern. J. Bacteriol. 183, 2605-2613.

Zhang, C.C., Laurent, S., Sakr, S., Peng, L., Bédu, S., 2006. Heterocyst differentiation 
and pattern formation in cyanobacteria: a chorus of signals. Mol. Microbiol. 59, $367-375$.

Zhou, R., Wei, X., Jiang, N., Li, H., Dong, Y., 1998. Evidence that HetR protein is an unusual serine-type protease. Proc. Natl. Acad. Sci. U S A 95, 4959-4563. 


\section{Figure Captions}

\section{Figure 1. The heterocyst pattern formation of Anabaena sp. PCC 7120}

under nitrogen-deprived culture. (A) Schematic representation of the morphological change in an Anabaena filament. In nitrogen-rich conditions, all cells along the filament are photosynthetic (vegetative). In nitrogen-depleted conditions, approximately every tenth vegetative cell differentiates into a nitrogen-fixing heterocyst. (B) Schematic representation of metabolic cooperation between a vegetative cell and heterocyst. Heterocysts supply the surrounding vegetative cells with fixed nitrogen compounds and receive carbohydrate products in return. (C) Photomicrographs of the heterocyst pattern. Heterocysts manifest as expanded cells with degraded phycobilisome proteins (a photosystem II light harvesting complex). Left and right panels display a bright field micrograph and a phycobilisome fluorescence micrograph, respectively. White arrows indicate heterocysts. Scale bar is $10 \mu \mathrm{m}$.

\section{Figure 2. Distributions of duration time of vegetative cells after latest cell} division at sampling times. (A) These panels show the distributions of duration time at 30, 35, 40, 50, 60, 70 hours a.n.d. We scored 40 cells at 30 hours, 48 cells at 35 hours, 71 cells at 40 hours, 92 cells at 45 hours, 132 cells at 50 hours, 174 cells at 55 hours, 235 cells at 60 hours, 329 cells at 65 hours, and 404 cells at 70 hours a.n.d. If a cell was dividing at any sampling time, the duration time of both sibling cells was scored as 0 hour. In the distributions at 30 and 35 hours a.n.d., we defined the duration time as hours after nitrogen depletion for the cell which had not divided (indicated by black arrows). (B) A scatter plot of the PC1 and PC2 scores obtained from principal 
component analysis (PCA). We note that the data at 30 hours a.n.d. include many cells which had not divided in the observation. Each point corresponds to the scores calculated from the distribution at the sampling times. The (PC1, PC2) scores were $(-0.249,0.301)$ at 30 hours, $(-0.253,-0.079)$ at 35 hours, $(-0.311,-0.053)$ at 40 hours, $(-0.285,-0.026)$ at 45 hours, $(-0.319,-0.0275)$ at 50 hours, $(-0.285,-0.020)$ at 55 hours, $(-0.281,0.029)$ at 60 hours, $(-0.312,-0.0395)$ at 65 hours, and $(-0.285,-0.043)$ at 70 hours a.n.d.

Figure 3. Distributions of cell cycle length in the generations 0-4 after nitrogen depletion. (A) In the generations $0,1,2,3$ and 4, the cell cycle length was scored from 24, 48, 91, 171, and 105 cells, respectively. All live, non-differentiated cells along the filament were evaluated. (B) A scatter plot of the PC1 and PC2 scores obtained from principal component analysis (PCA). Each point corresponds to the scores calculated from the distribution in each generation. The (PC1, PC2) scores for generations $1,2,3$ and 4 were $(-0.309,-0.173),(-0.360,0.0464),(-0.364,0.0547)$, and $(-0.359,0.0475)$, respectively. (C) The steady-state distribution of cell cycle length is the sum of the frequency distributions of generations 2-4.

\section{Figure 4. Distributions of segment length observed at 65, 90, 185 hours}

a.n.d. Means and standard deviations, respectively, were (A) 9.11 and 2.93 (from 166 segments) at 65 hours a.n.d. (B) 11.35 and 3.85 (from 164 segments) at 90 hours a.n.d. (C) 11.11 and 3.81 (from 159 segments) at 185 hours a.n.d. No contiguous heterocysts or segment length exceeding 30 cells were observed at any time. 
Figure 5. Schematic representation of the transition process among cell division, differentiation and maturation. As the cell matures, its cell age increases by one step. Once a cell divides, the cell age of both daughter cells are initialized to 1. A differentiated cell undergoes no further dynamical change. If cell division and differentiation are considered to depend on the cell age, older cells will more likely divide or differentiate than younger cells. Here, $i$ denotes the cell age. $p(i), q(i)$, and $r(i)$ are the division, differentiation, and maturation rates, respectively.

Figure 6. Distributions of cell division and differentiation time length, extracted from experimental data. (A) A distribution of cell cycle length as a function of cell age. The frequencies of cell cycle length are plotted as red dots. The frequencies are well-fitted to a Hill function (solid black line). (B) A later-stage distribution of time length from the latest cell division to the onset of differentiation, as a function of the cell age. The frequencies of time length are plotted as red dots. Again, these frequencies satisfy a Hill function (solid black line). In both plots, the values of cell age are standardized to $\in[1,10]$ a.u. from time length data $\in[1,13] \mathrm{h}$.

Figure 7. Schematic representation of the transition process of segment length. Cell division of any vegetative cell increases the segment length by 1 . Differentiation of any vegetative cell divides the segment into two shorter segments (indicated by red arrows). The dark green cells flanking the segment are heterocysts; the light green intermediate cells are vegetative cells. $l(n)$ is the lateral inhibition rate ( $n$ is the number of vegetative cells between a focal cell and each heterocyst in a segment). $\hat{p}$ and $\hat{q}$ are the average division and differentiation rates, respectively. 
Figure 8. Representative comparisons between the analytical solution and numerical distribution of segment length. The same kinetic parameter values were used in the analytical solution (red line) and numerical simulation (blue line). In Eq. (4), the maximum segment length $n$ was set to 50 . Values of $(a, b, c, d, e, f)$ were (A) (0.3, $0.3,0.4,0.8,10,6)$; (B) $(0.3,0.3,0.4,1.6,20,6)$; (C) $(0.3,0.3,0.4,0.6,5,6)$; (D) $(0.1$, $0.23,0.25,0.8,10,6)$.

Figure 9. Comparison between the analytical solution and experimental distribution of the later pattern. The analytical solution and experimental distribution are represented by the red and black histogram, respectively. The analytical solution assumed the optimal kinetic parameter values $((a, b, c, d, e, f)=(0.06,0.7,0.25,0.8,10$, 6)), as estimated by our mathematical method. The mean (standard deviation) of the analytical solution and experimental distribution are 11.28 (3.78) and 11.35 (3.85), respectively.

Figure 10. Sensitivity analysis investigating the dependency of later pattern formation on the estimated kinetic parameters. Each parameter value was varied while fixing the other five parameters with their estimated values. The investigated range of each kinetic parameter was within the range selected to optimize the parameter values. Histograms show the posterior probabilities of each parameter value. In each histogram, the highest posterior probability at which the kinetic parameter value matches the estimated one is indicated by a red arrow. 
Figure 11. Analytically determined distributions of cell age during the early

stage. Blue and red dots indicate the cell age frequencies when differentiation depends on the cell age $((a, b, c, d, e, f)=(0.01,0.6,0.75,0.8,10,6))$ and when it is independent of the cell age $((a, b, c, d, e, f)=(0.06,0.7,0.25,0.8,10,6))$, respectively.

Figure 12. Transition of hetR::gfp fluorescence signal ratios before or after cell division. The fluorescence signal ratios before or after cell division to that at 1-hour before cell division (average $\pm \mathrm{SD}$ ) are calculated as mentioned in Appendix G. Panel A and B show the transition of fluorescence signal ratios of cells, which divided at 63-65 hours and 69-70 hours a.n.d., respectively. Gray zones indicate the early (A) or later (B) stages. Black arrows indicate the time of cell division.

Figure 13. Sensitivity analysis investigating the dependency of early pattern formation on the estimated kinetic parameters. (A) Each kinetic parameter value $a$, $b, c$ was varied while the remaining two parameters were fixed at their estimated values. The kinetic parameters $d, e, f$ were assigned the same values as in the later pattern formation. (B) The kinetic parameter value $a$ was varied while fixing the other five parameters $(b, c, d, e, f)$ at $(0.7,0.25,0.8,10,6)$. The investigated range of each parameter was within the range selected to optimize the parameter values. Histograms indicate the posterior probabilities of each parameter. In each histogram, the highest posterior probability at which the kinetic parameter value matches the estimated one is indicated by a red arrow. 
Figure 14. Comparison between the analytical solution and experimental distribution of the early pattern. The analytical solution and experimental distribution are represented by the red and black histogram, respectively. The analytical solution assumed the same kinetic parameter values as the later pattern $((a, b, c, d, e, f)=(0.06$, $0.7,0.25,0.8,8,6)$ ). The mean (standard deviation) of the analytical solution and experimental distribution are 9.44 (3.14) and 9.11 (2.93), respectively.

Figure G1. Schematic representation of cells before or after cell division (sample line). The mother cell before cell division is termed as " $m$ ", while the two sibling cells after cell division are termed as " $s 1$ " and " $s 2$ ", respectively. The time (hour) before or after cell division is indicated below each cell. 
Table Captions (for the Appendix C)

Table C1 $p$-values among the distributions of duration time after cell division.

$p$-values among the distributions of duration time after latest cell division were calculated by two-sample two-sided Kolmogorov-Smirnov tests.

Table C2 p-values among the distributions of cell cycle length.

$p$-values among the distributions of cell cycle length in the generations $2-4$ were calculated by two-sample two-sided Kolmogorov-Smirnov tests. 
Table (for the Appendix C)

Table C1 $p$-values among the distributions of duration time after cell division.

\begin{tabular}{|c|c|c|c|c|c|c|c|c|}
\hline & $35 \mathrm{~h}$ & $40 \mathrm{~h}$ & $45 \mathrm{~h}$ & $50 \mathrm{~h}$ & $55 \mathrm{~h}$ & $60 \mathrm{~h}$ & $65 \mathrm{~h}$ & $70 \mathrm{~h}$ \\
\hline $30 \mathrm{~h}$ & $9.26 \times 10^{-3}$ & 0.0622 & $8.64 \times 10^{-3}$ & $4.16 \times 10^{-3}$ & $5.26 \times 10^{-3}$ & $9.56 \times 10^{-3}$ & $8.12 \times 10^{-4}$ & $7.56 \times 10^{-3}$ \\
\hline $35 \mathrm{~h}$ & & 0.0101 & 0.354 & 0.0112 & 0.118 & 0.0354 & 0.0119 & 0.155 \\
\hline $40 \mathrm{~h}$ & & & 0.332 & 0.494 & 0.128 & 0.329 & 0.568 & 0.0690 \\
\hline $45 \mathrm{~h}$ & & & & 0.0432 & 0.2026 & 0.124 & 0.0119 & 0.750 \\
\hline $50 \mathrm{~h}$ & & & & & 0.205 & 0.559 & 0.524 & 0.0806 \\
\hline $55 \mathrm{~h}$ & & & & & & 0.735 & 0.0429 & 0.133 \\
\hline $60 \mathrm{~h}$ & & & & & & & 0.109 & 0.045 \\
\hline $65 \mathrm{~h}$ & & & & & & & & 0.0113 \\
\hline
\end{tabular}

Table C2 p-values among the distributions of cell cycle length.

\begin{tabular}{|c|c|c|c|}
\hline & generation 2 & generation 3 & generation 4 \\
\hline generation 1 & $2.12 \times 10^{-3}$ & $1.15 \times 10^{-4}$ & $5.00 \times 10^{-4}$ \\
\hline generation 2 & & 0.999 & 0.999 \\
\hline generation 3 & & & 0.999 \\
\hline
\end{tabular}


A

Anabaena sp. PCC 7120

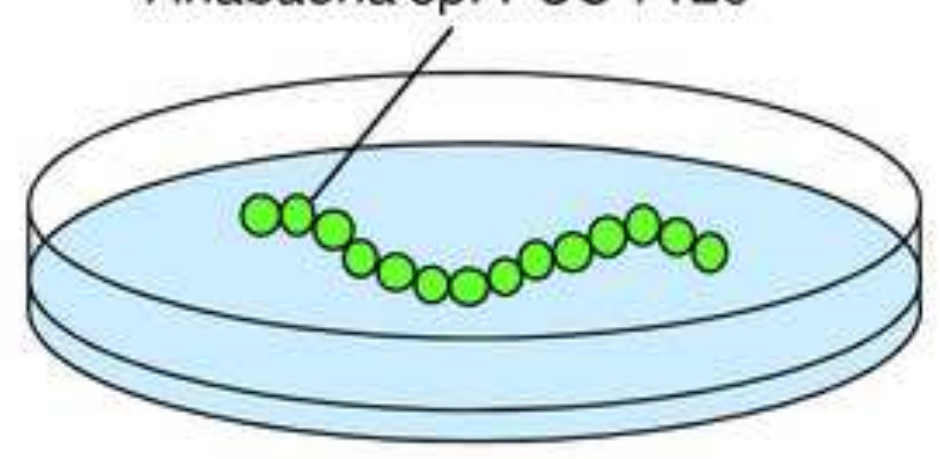

$\downarrow$ Under Nitrogen-deprived culture

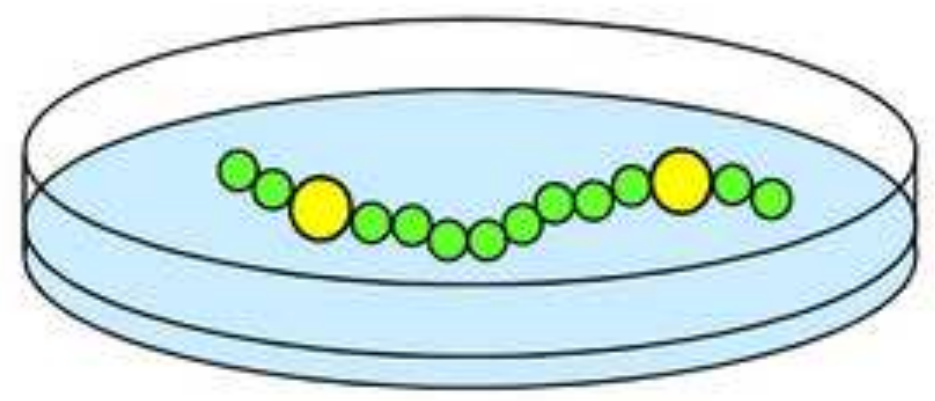

B
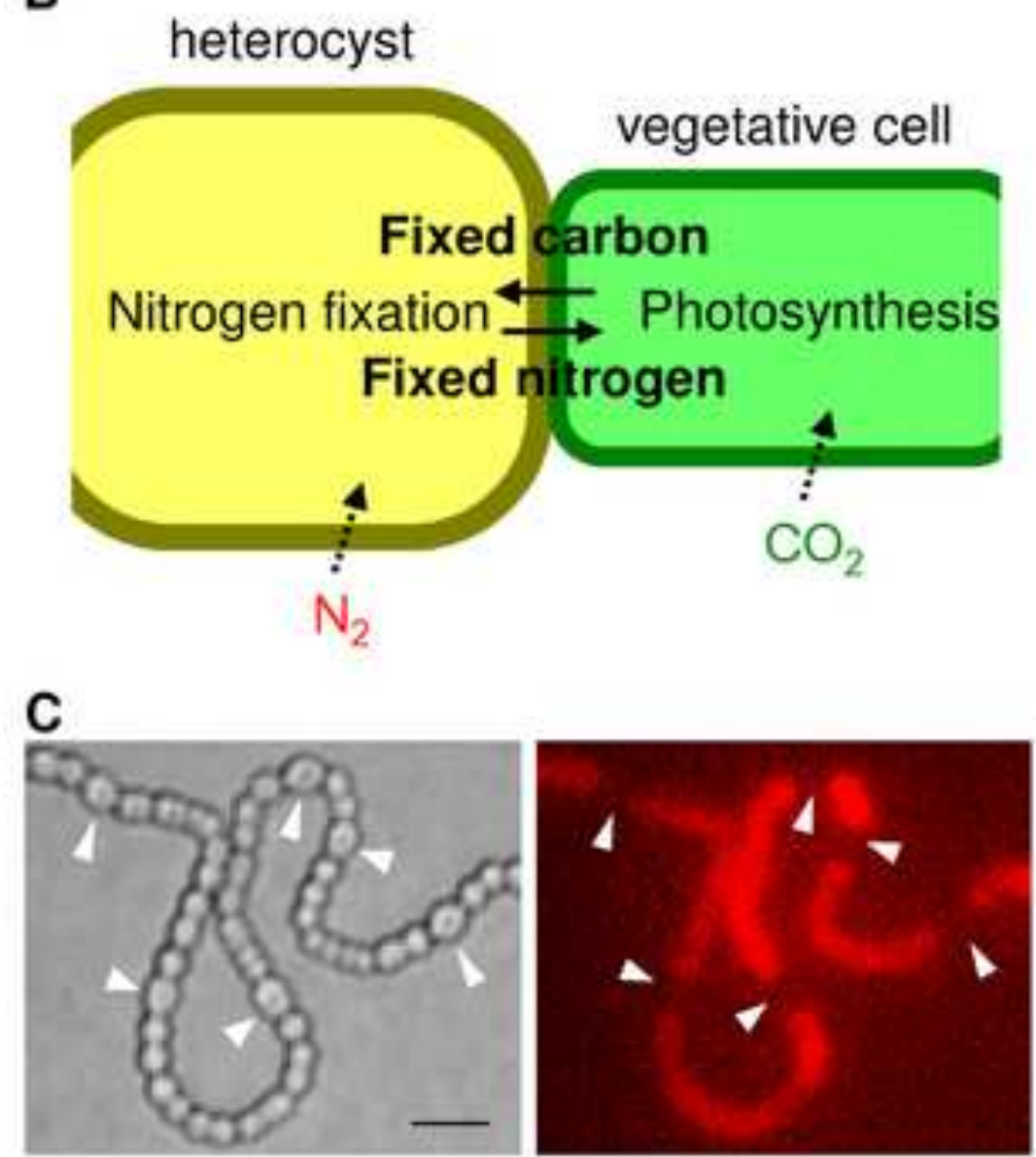
Figure 2

A

30 hours a.n.d.

35 hours an.d.

40 hours a.n.d.
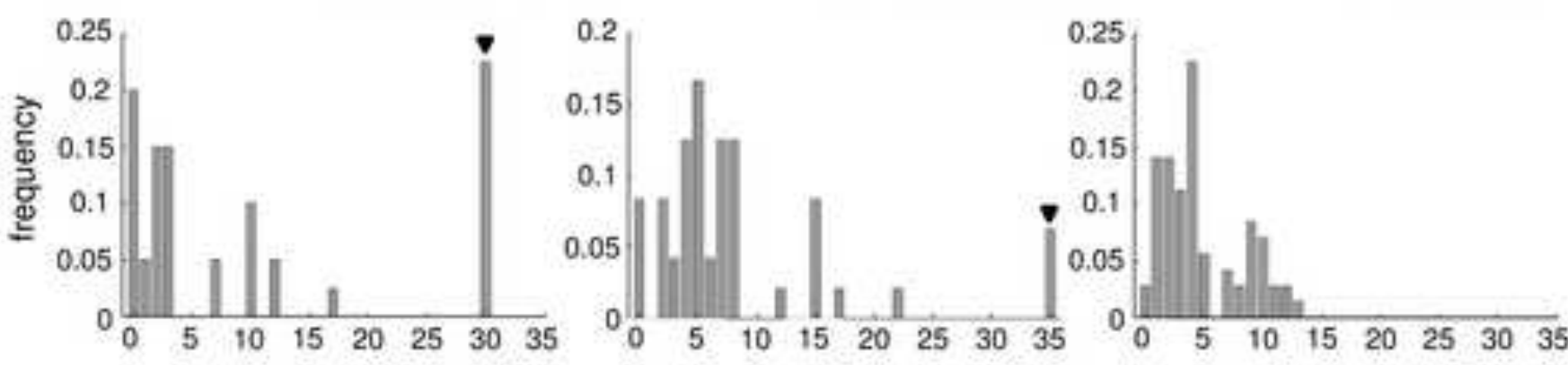

duration time after latest cell division at sampling time [h]
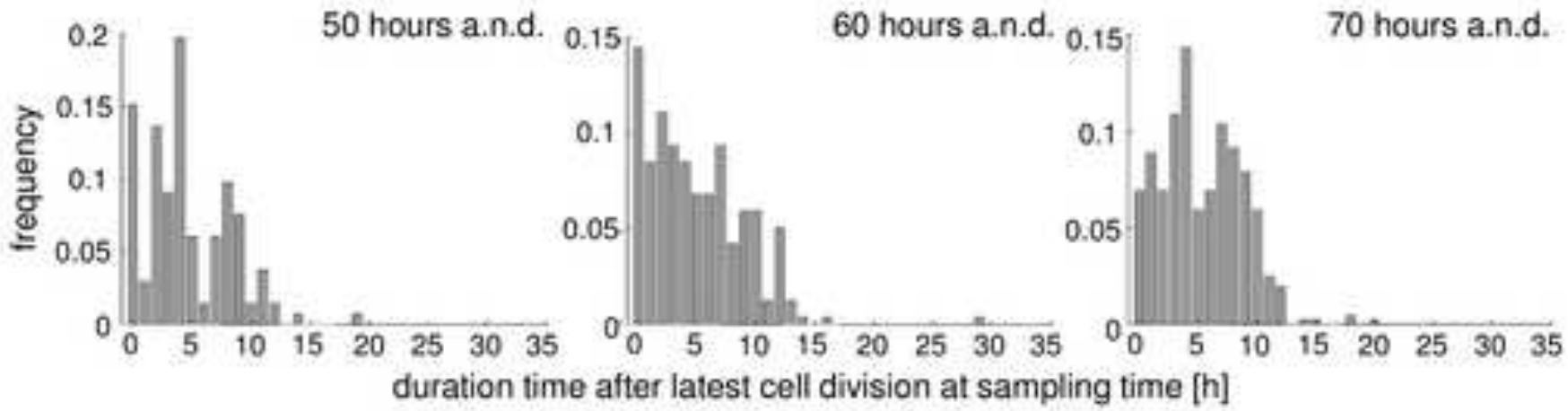

B

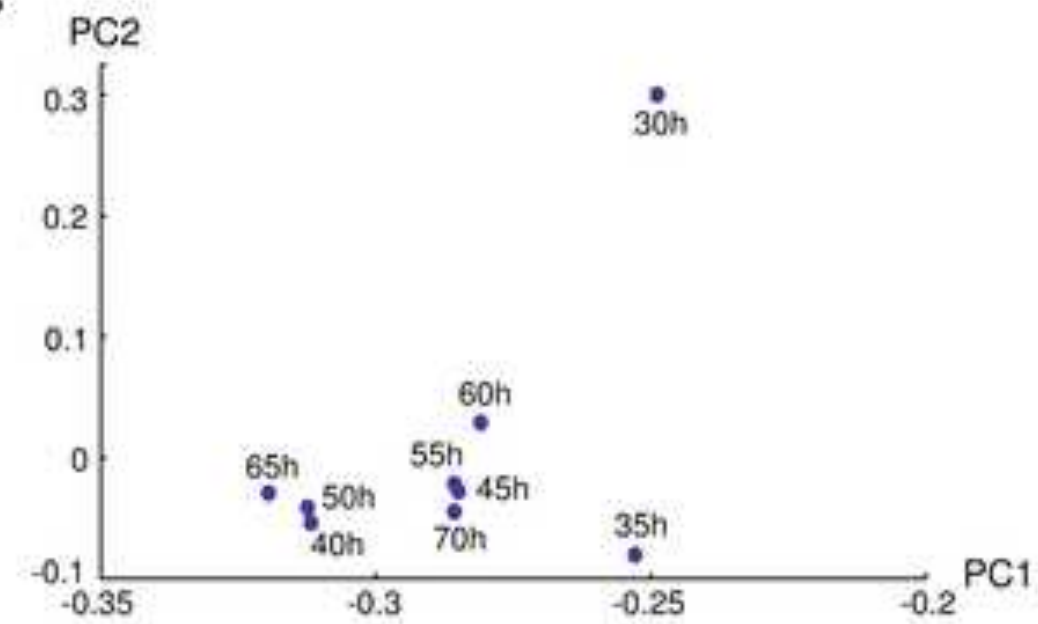


Figure 3 A
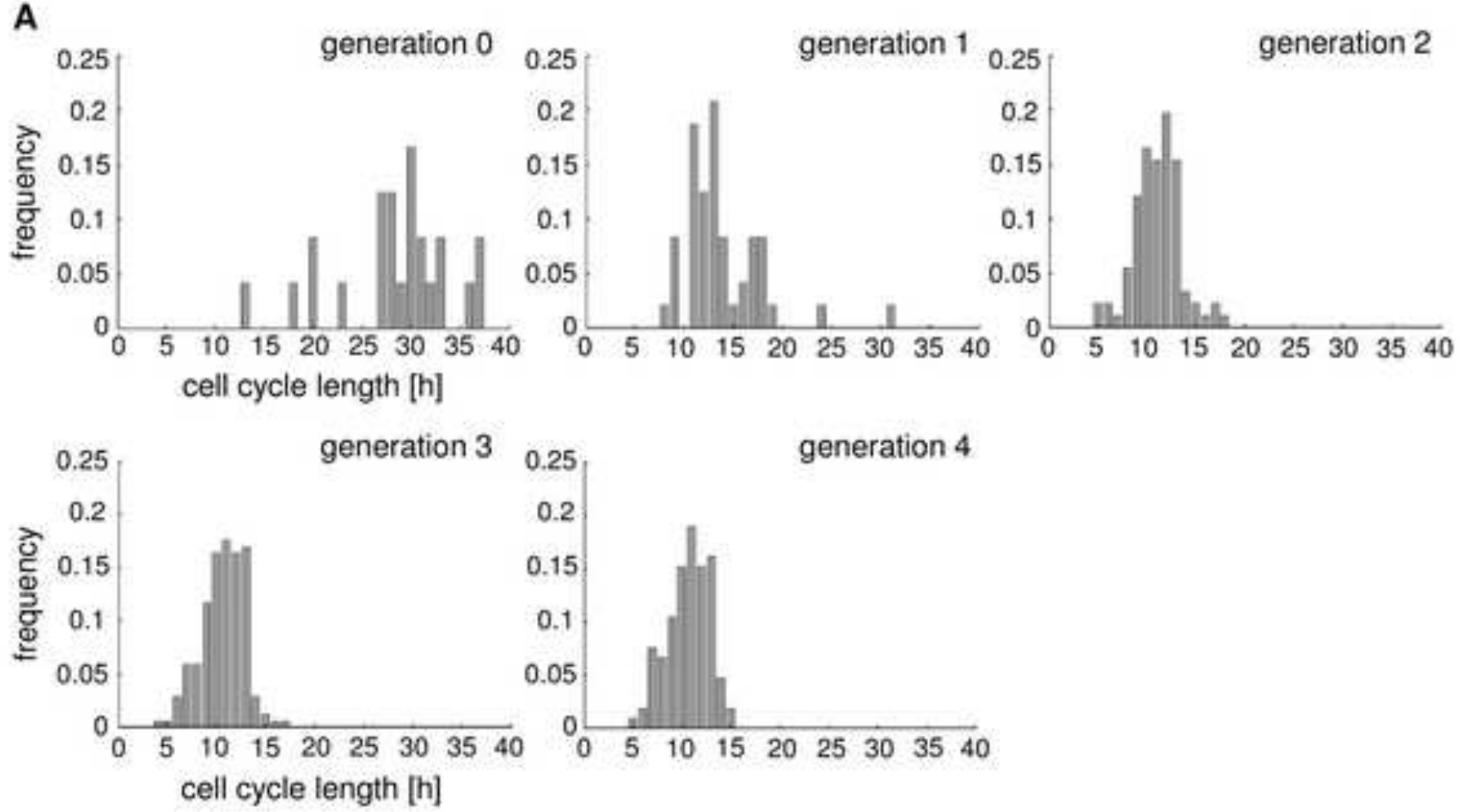

B
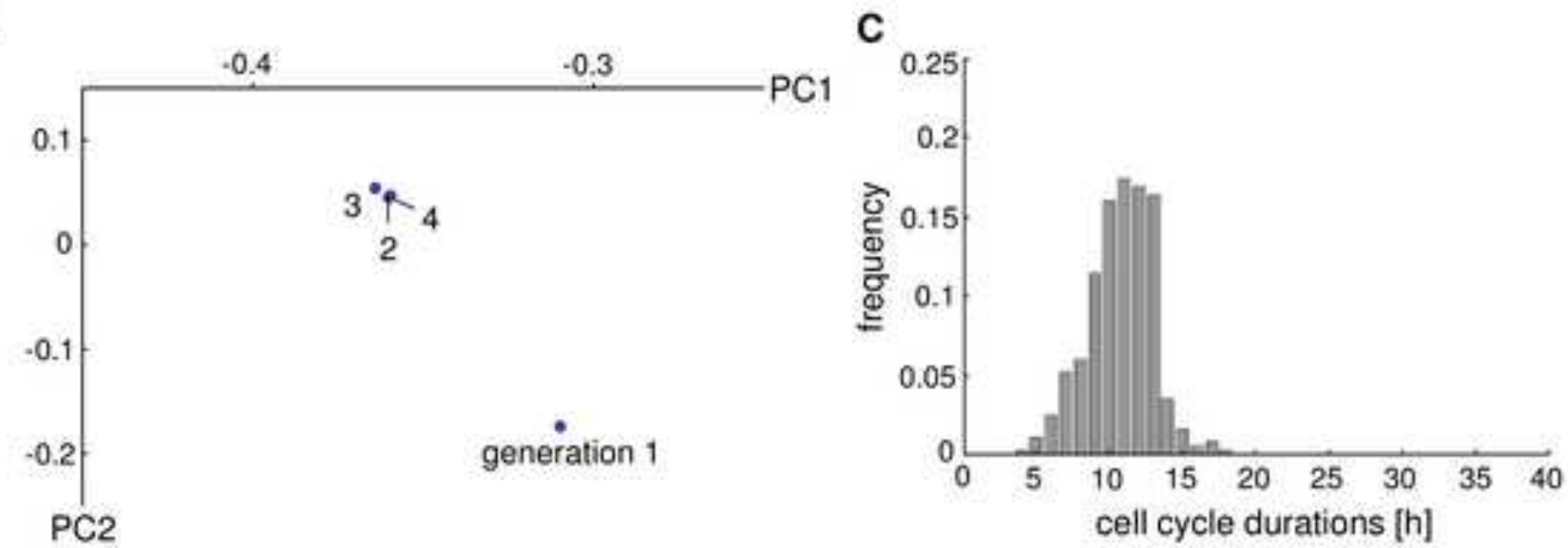
Figure 4

A
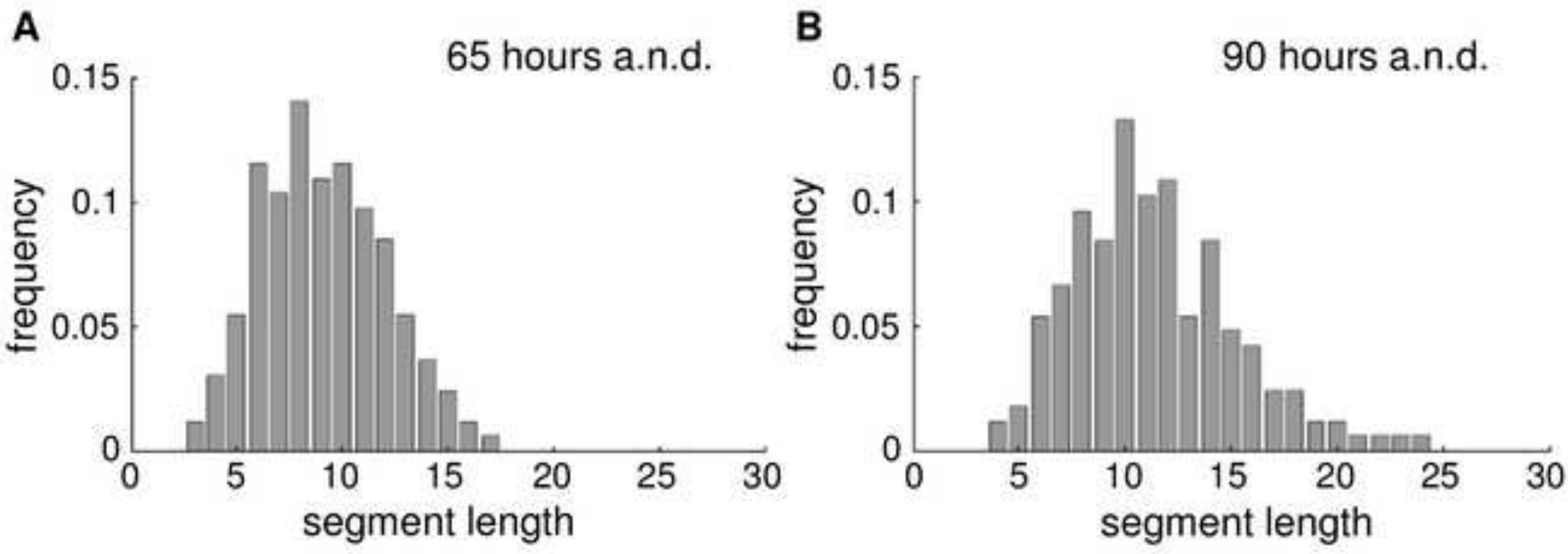

C

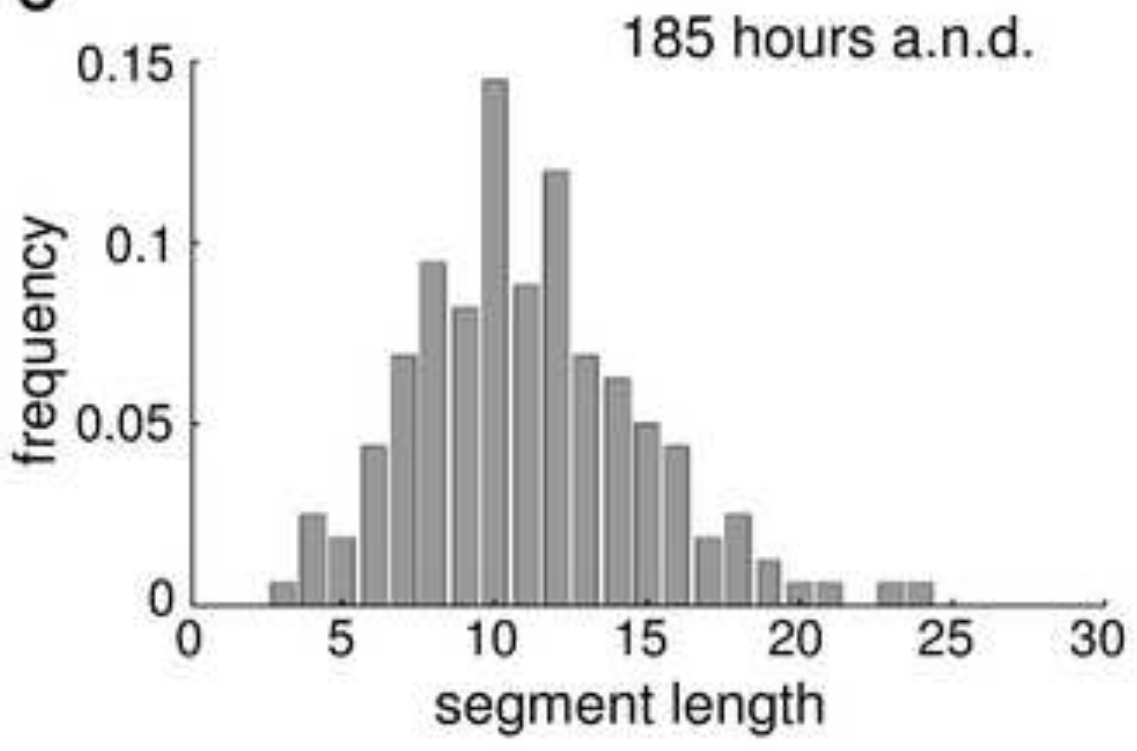


Figure 5

\section{cell division}

differentiation

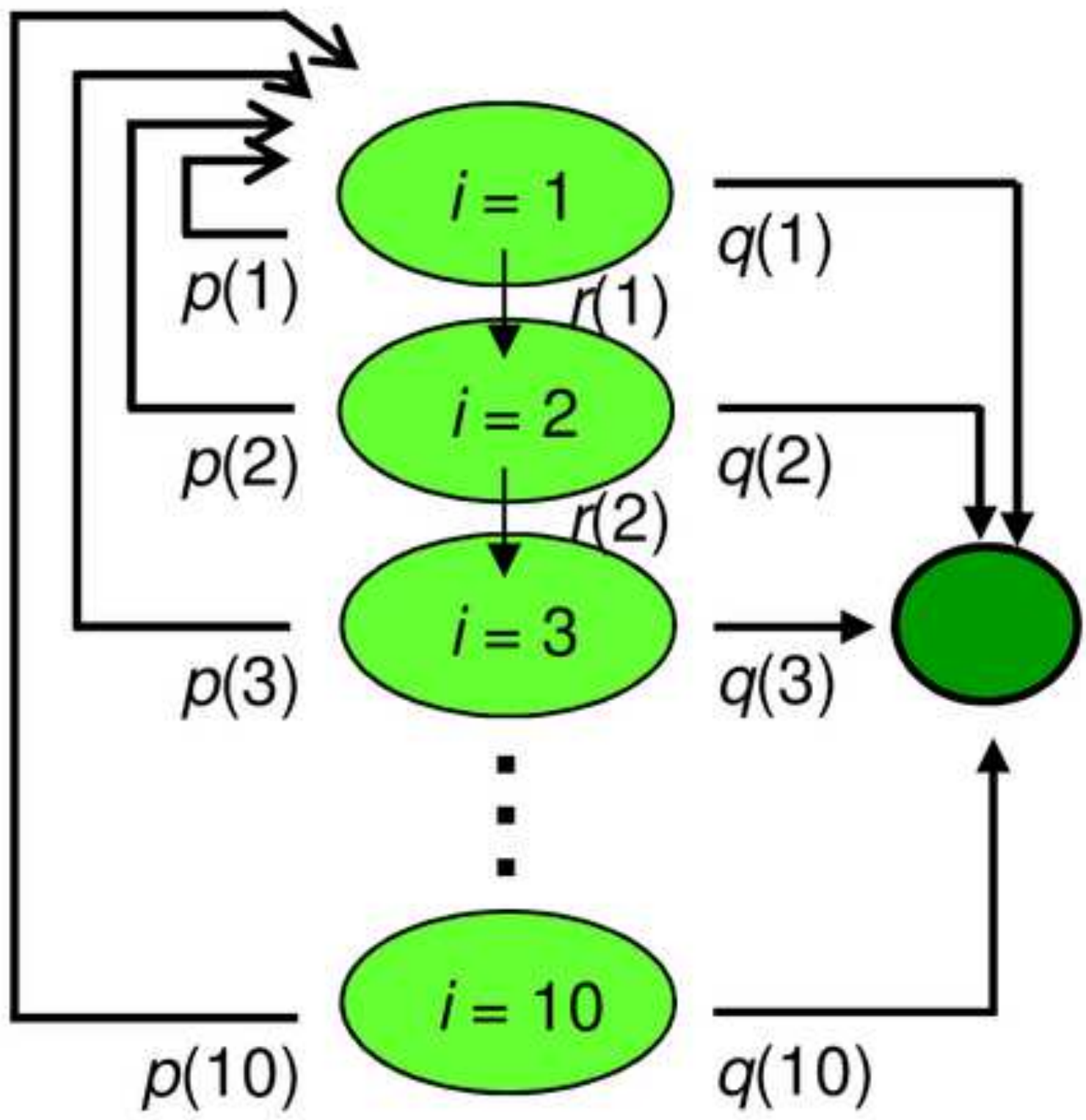


Figure 6

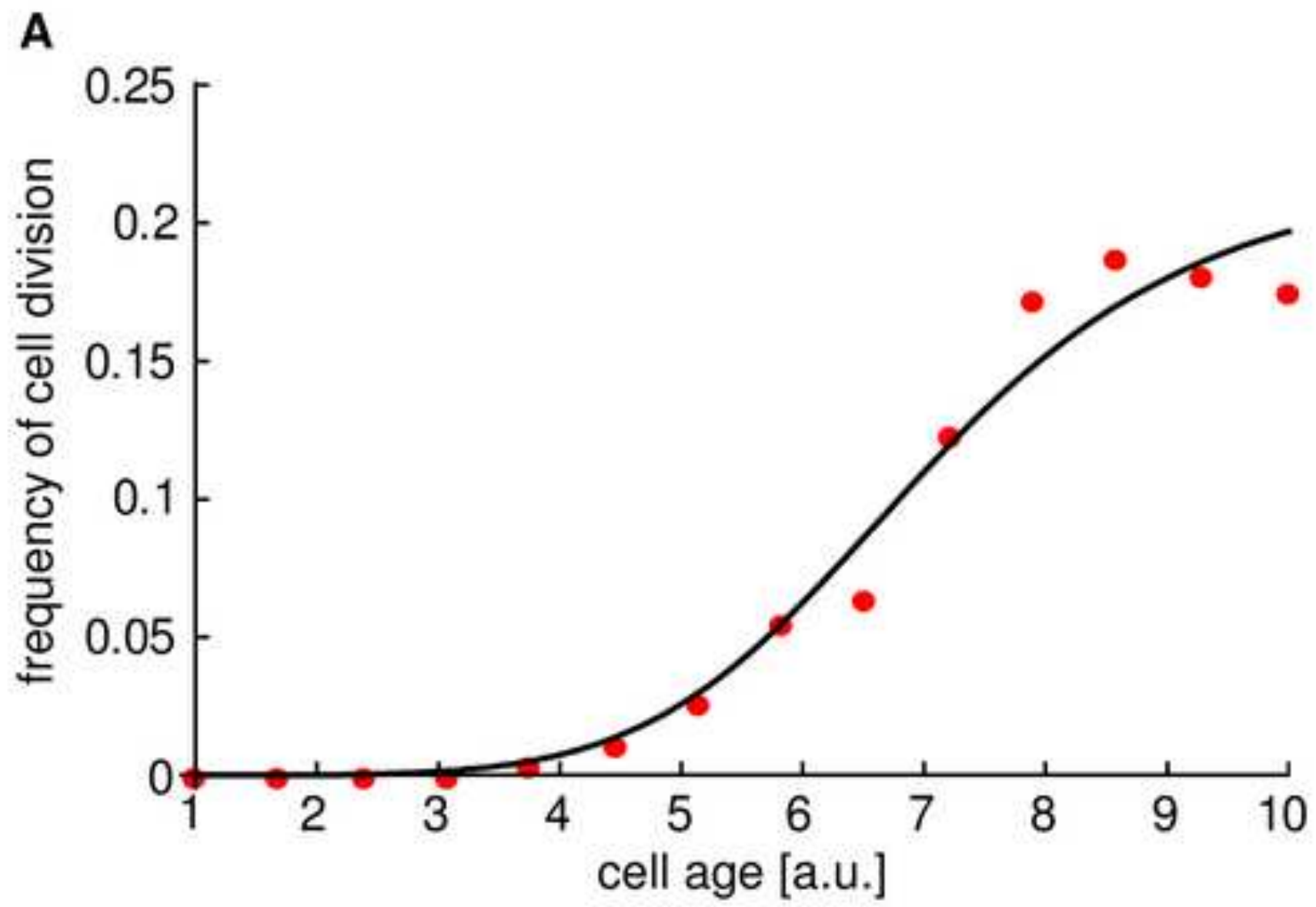

B

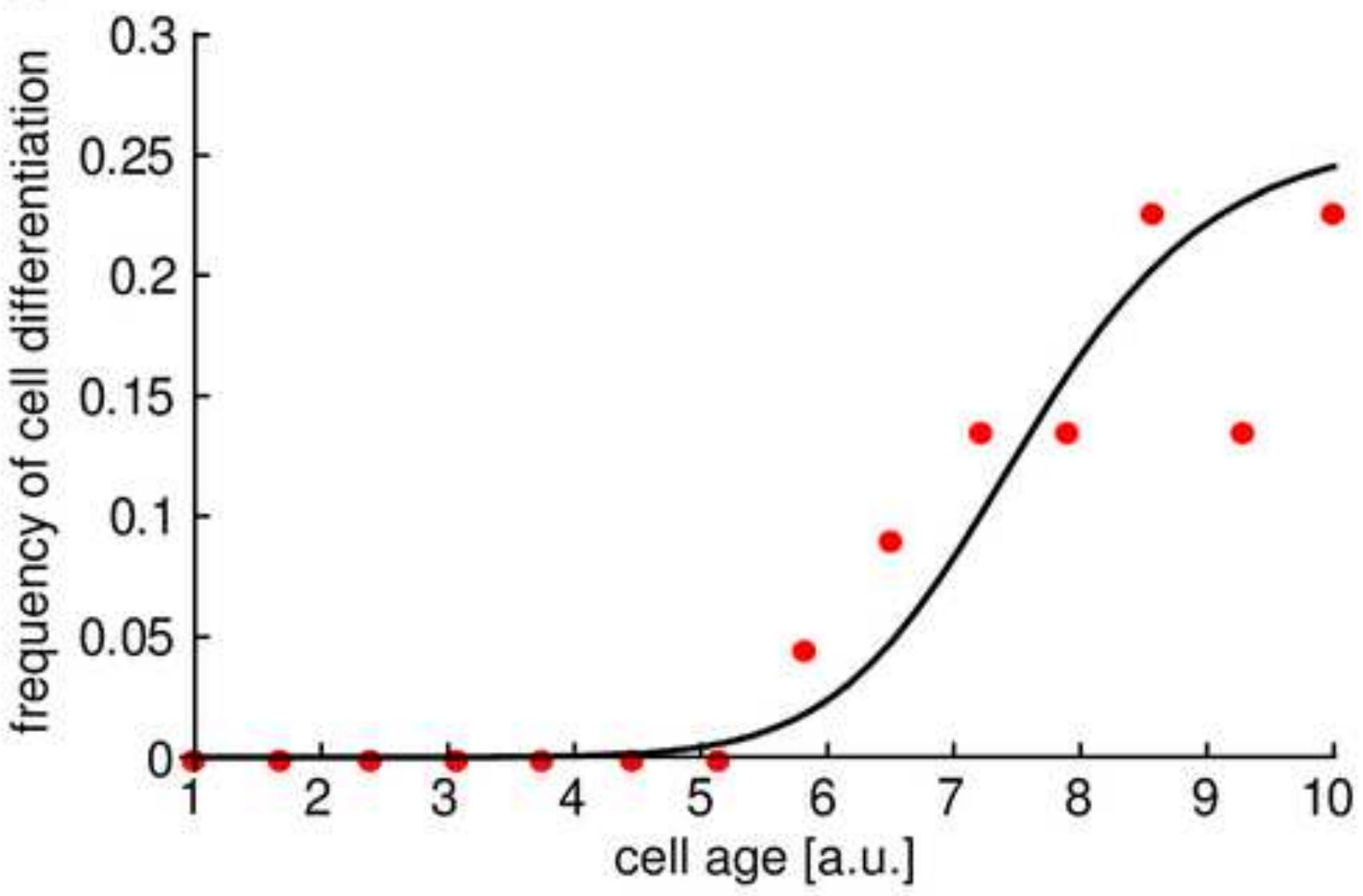


Figure 7

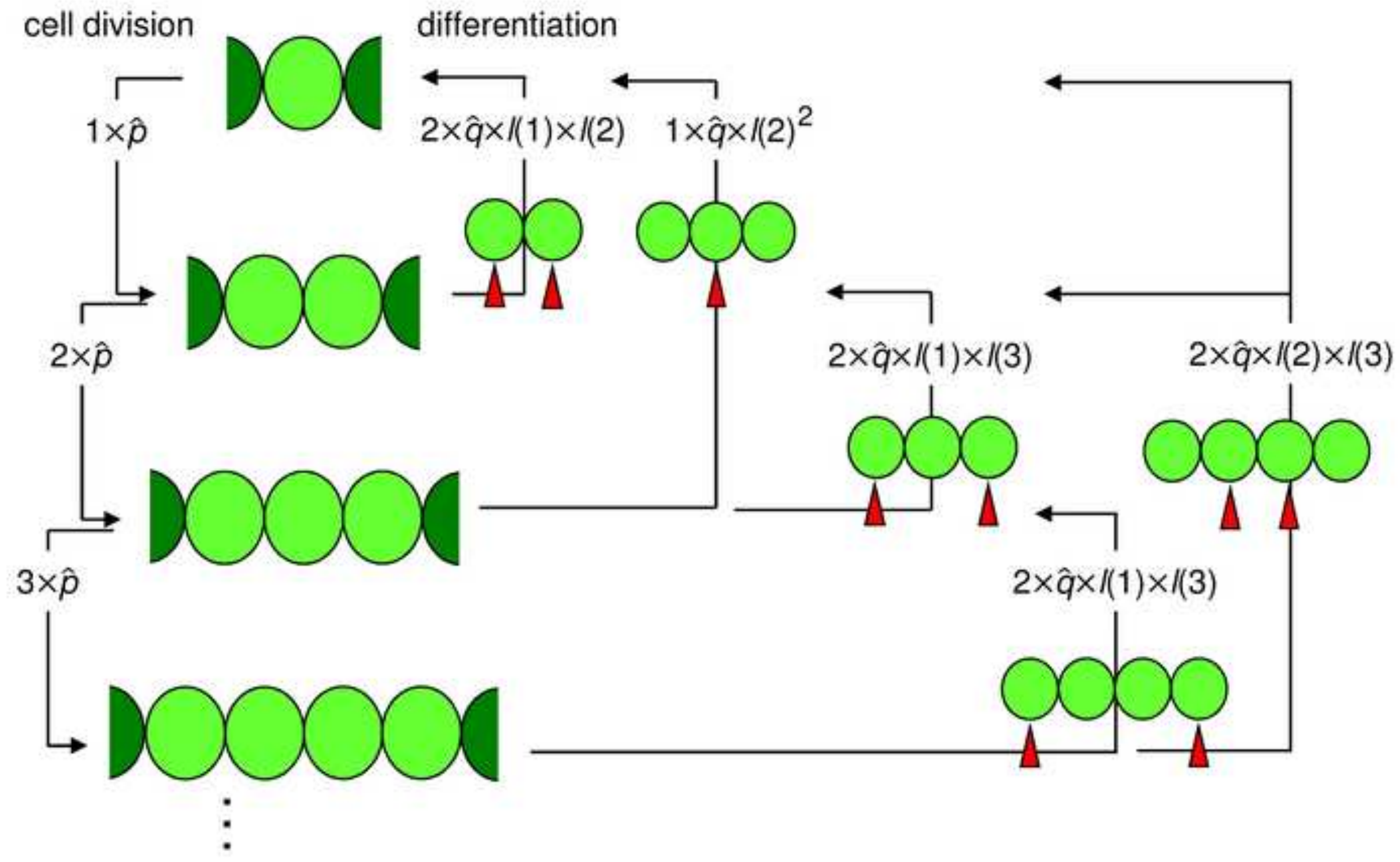


Figure 8
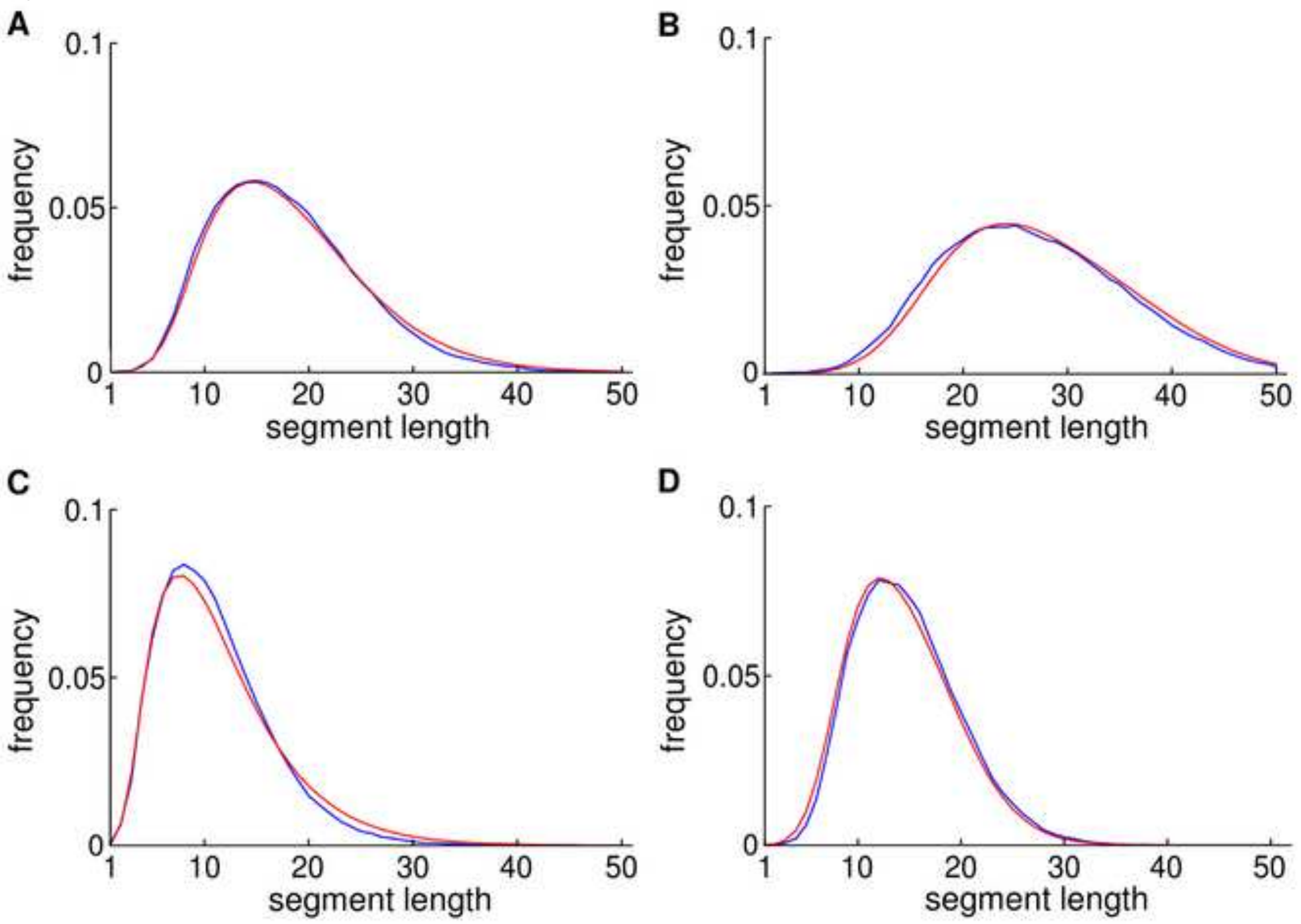
Figure 9

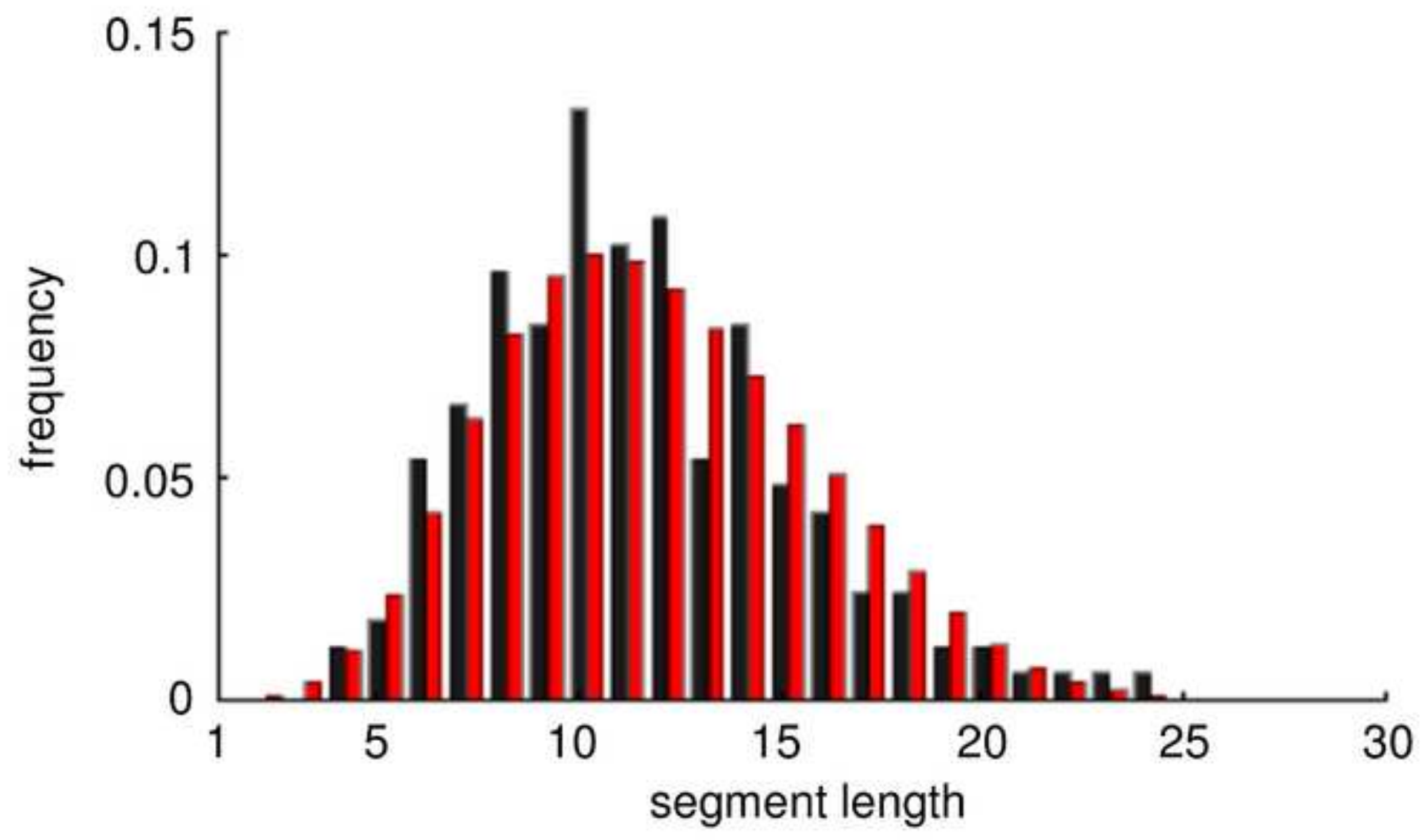


Figure 10
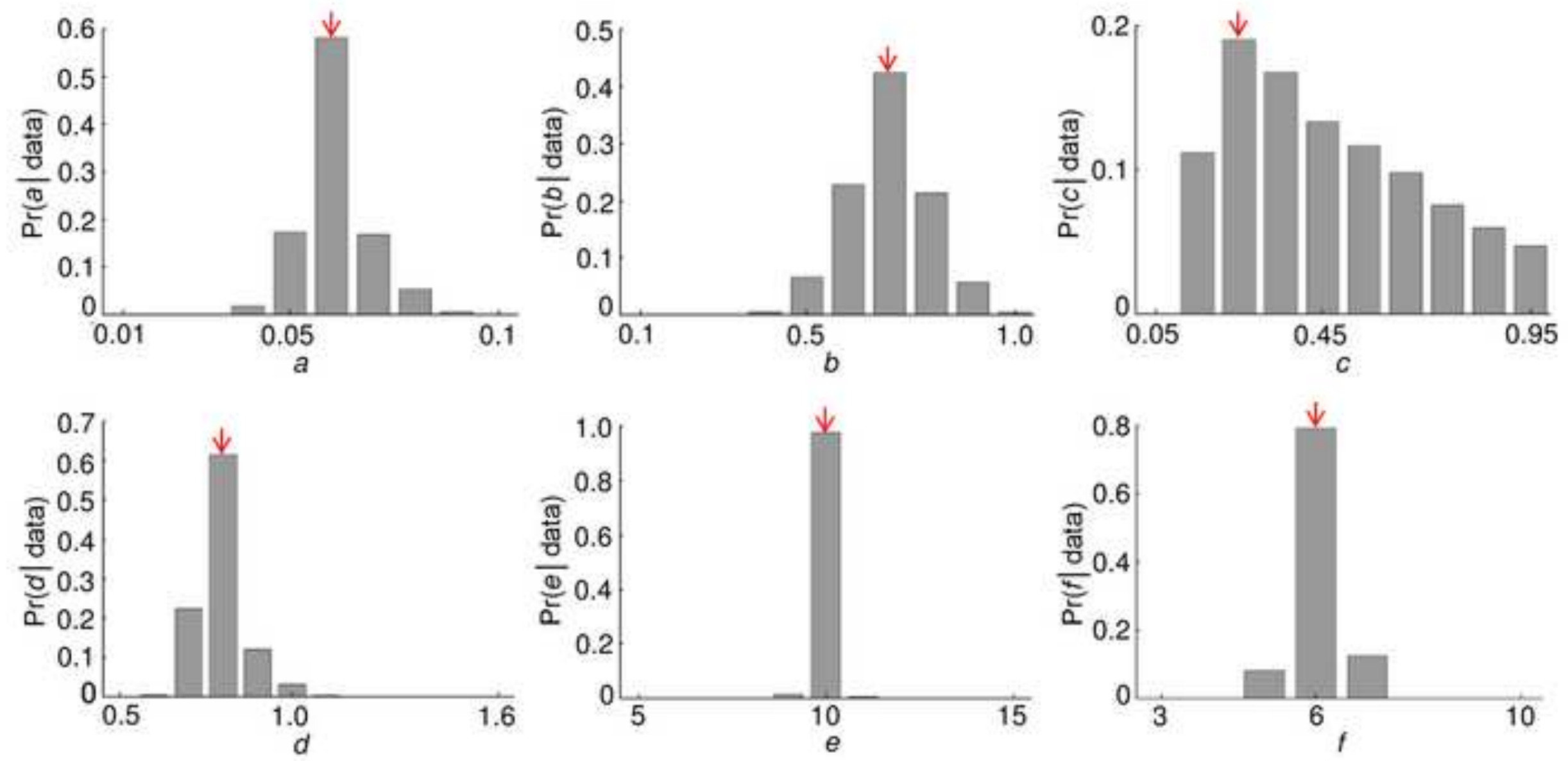
Figure 11

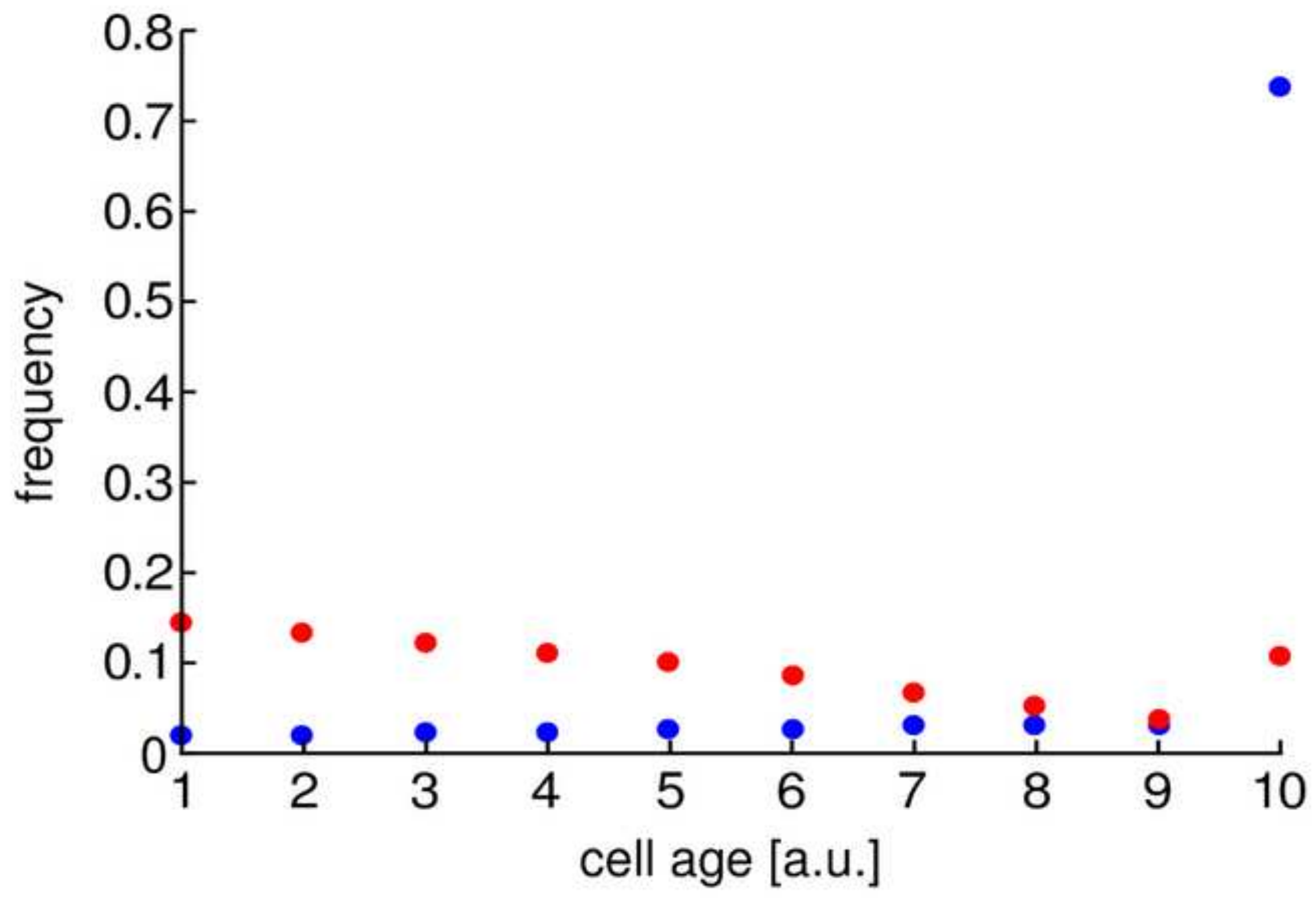


Figure 12
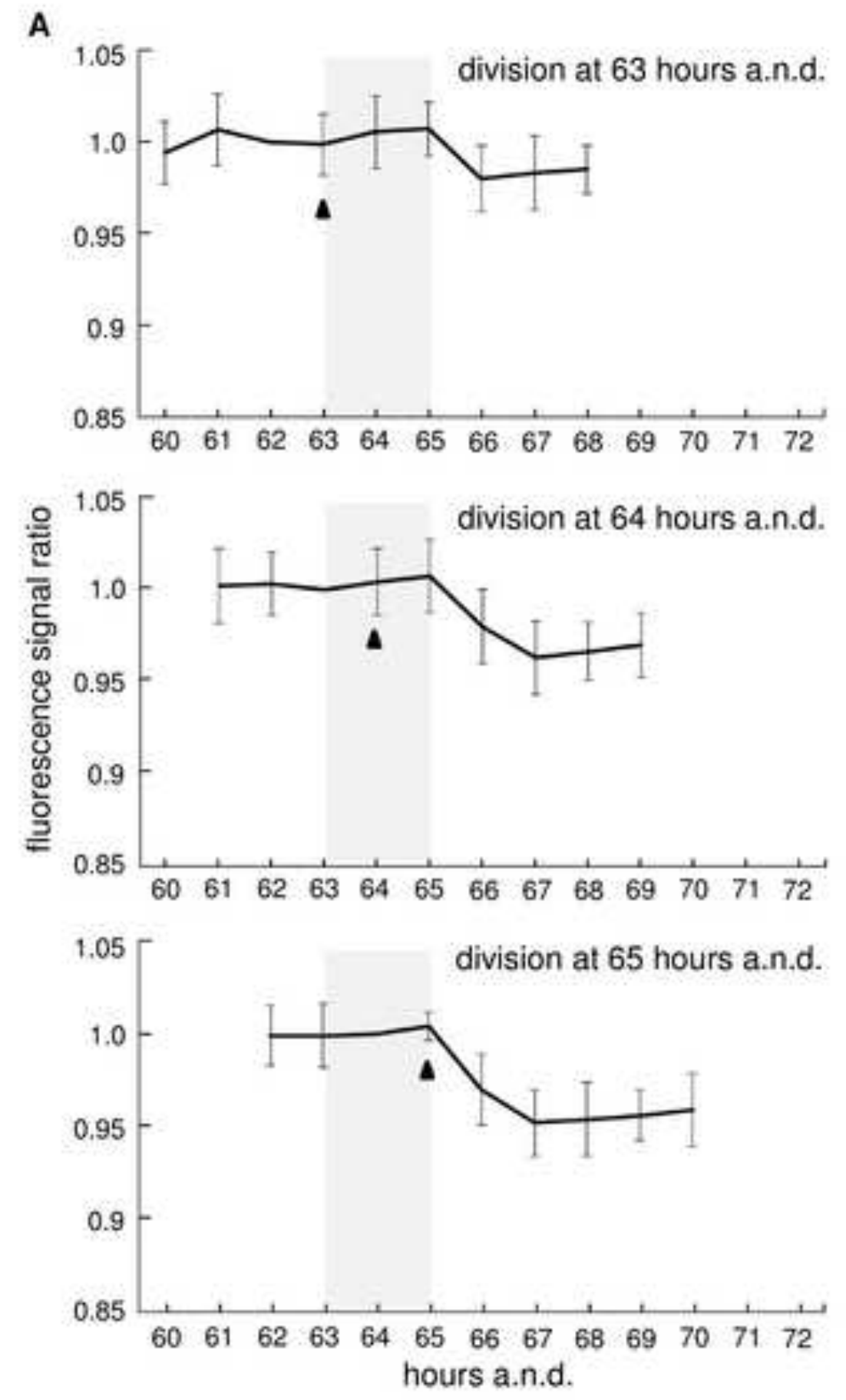

B

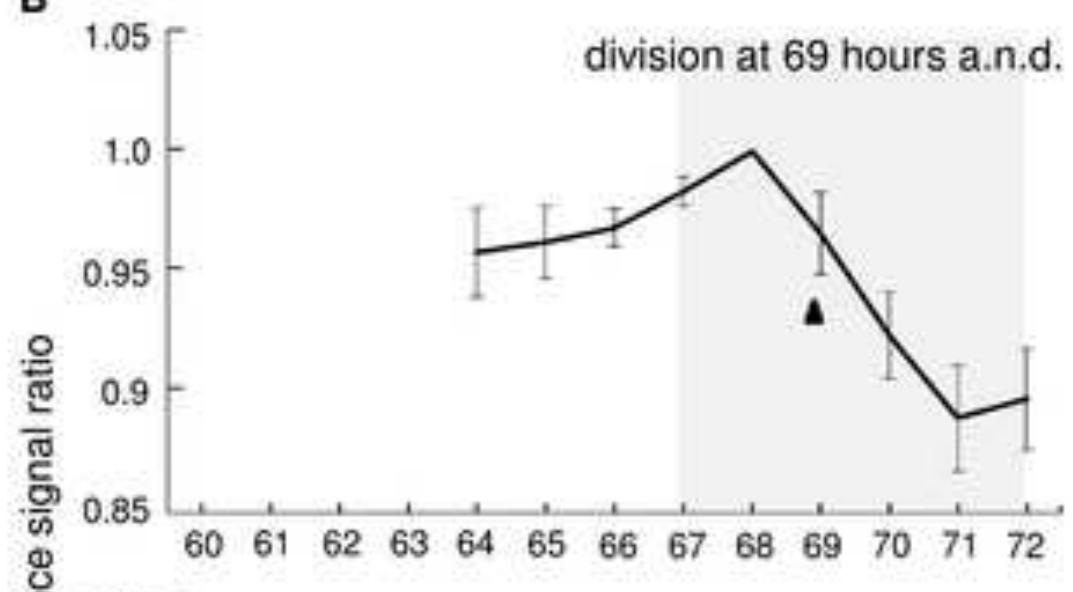

등

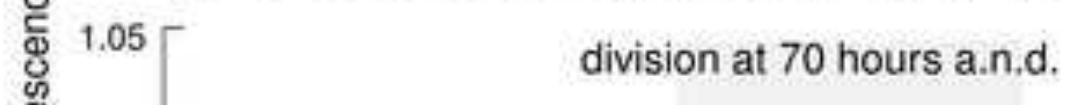

$1.0-$
0.95
0.9
0.85

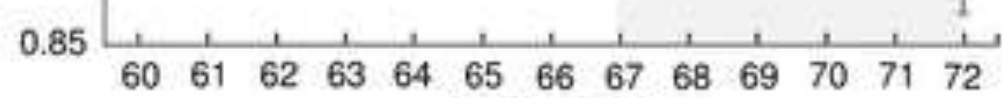
hours a.n.d. 
Figure 13
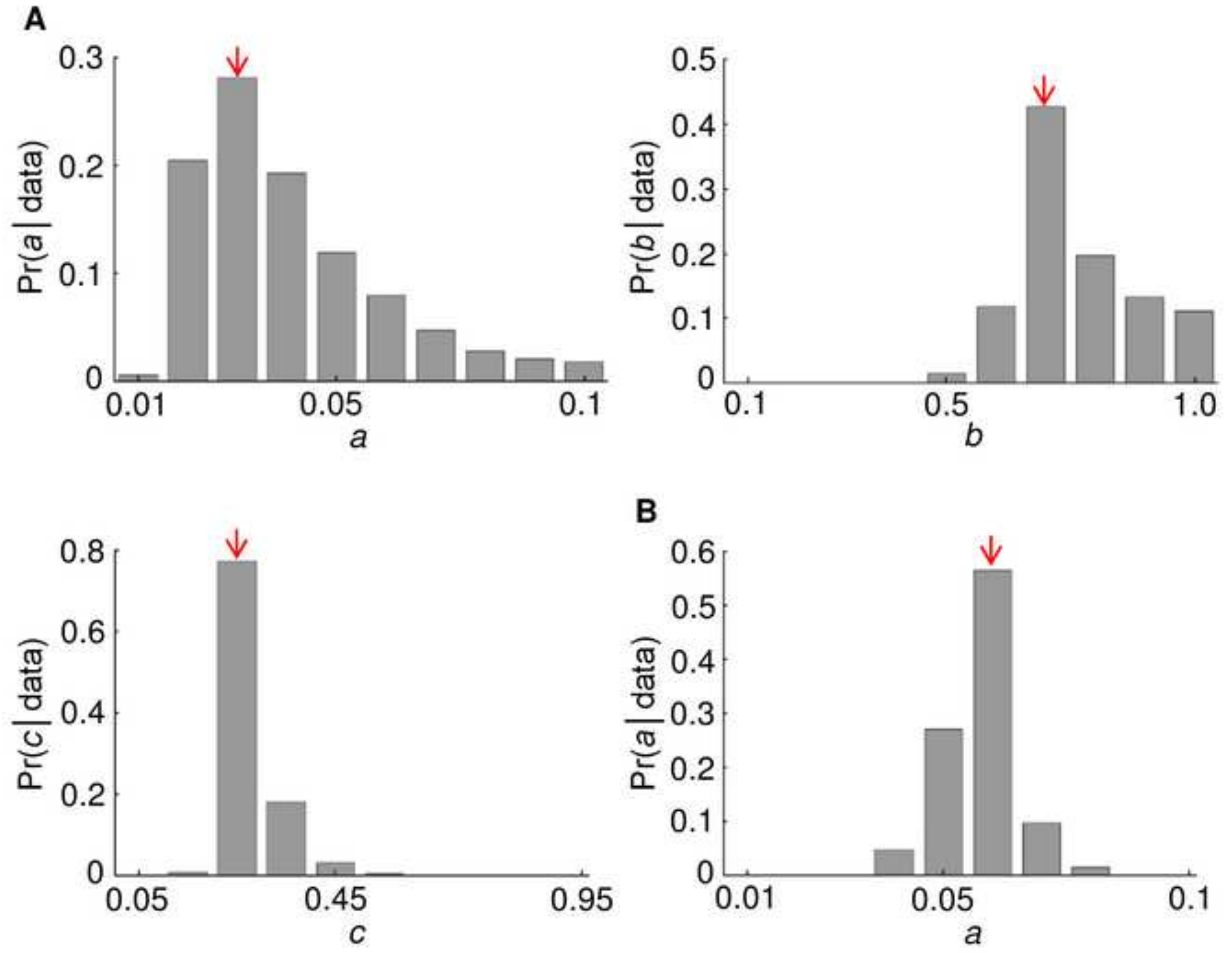
Figure 14

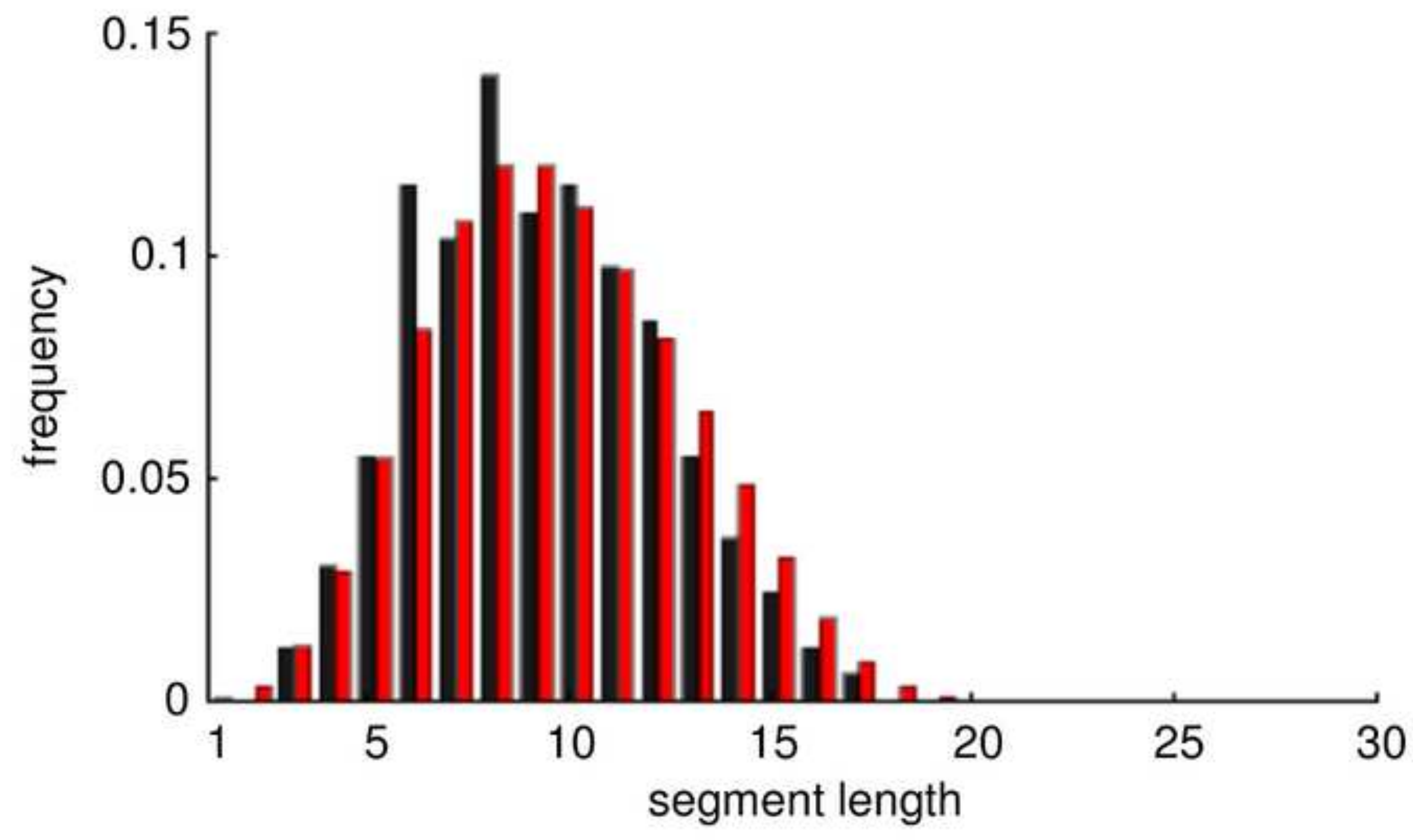




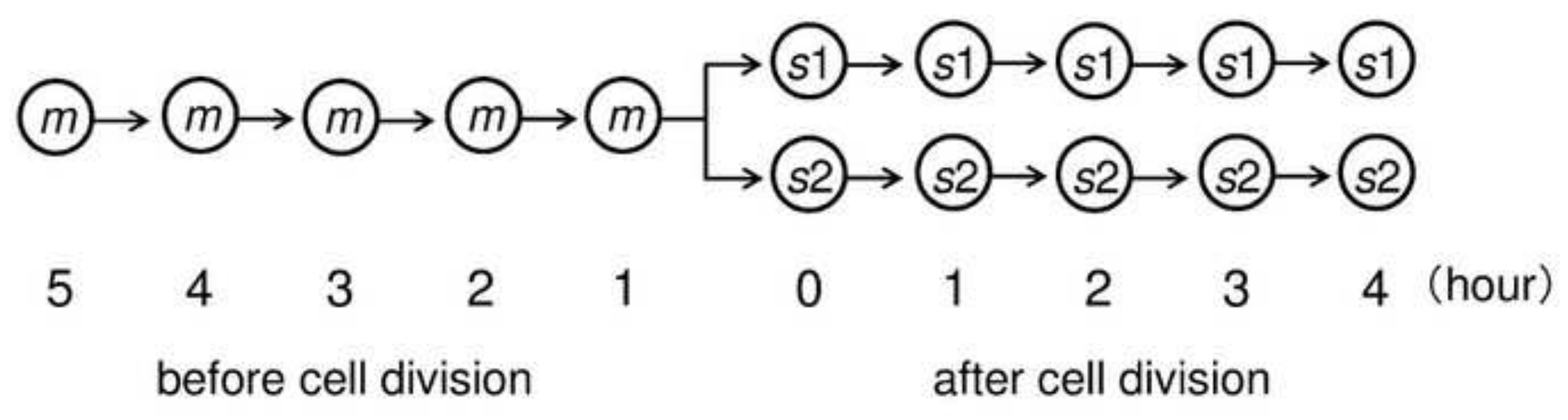

